\title{
Additive habit formation Consumption in incomplete markets with random endowments
}

\author{
Journal Article \\ Author(s): \\ Muraviev, Roman \\ Publication date: \\ 2011-09 \\ Permanent link: \\ https://doi.org/10.3929/ethz-b-000040571 \\ Rights / license: \\ In Copyright - Non-Commercial Use Permitted \\ Originally published in: \\ Mathematics and Financial Economics 5(2), https://doi.org/10.1007/s11579-011-0049-y
}




\title{
Additive habit formation: consumption in incomplete markets with random endowments
}

\author{
Roman Muraviev
}

Received: 6 May 2011 / Accepted: 11 July 2011 / Published online: 23 July 2011

(C) Springer-Verlag 2011

\begin{abstract}
We provide a detailed characterization of the optimal consumption stream for the additive habit-forming utility maximization problem, in a framework of general discrete-time incomplete markets and random endowments. This characterization allows us to derive the monotonicity and concavity of the optimal consumption as a function of wealth, for several important classes of incomplete markets and preferences. These results yield a deeper understanding of the fine structure of the optimal consumption and provide a further theoretical support for the classical conjectures of Keynes (The general theory of employment, interest and money. Cambridge University Press, Cambridge, 1936).
\end{abstract}

Keywords Utility maximization · Habit formation · Consumption · Incomplete markets · Random endowments

Mathematics Subject Classification (2000) $\quad 91 \mathrm{~B} 28 \cdot 60 \mathrm{~F} 15 \cdot 91 \mathrm{~B} 30$

JEL Classification $\mathrm{G} 11 \cdot \mathrm{E} 21$

\section{Introduction}

The class of habit-forming expected utility models have become increasingly popular in financial economics in recent years. While time-separable expected utility models are routinely found to be inconsistent with experimental evidence on choice under uncertainty (see [28]), habit preferences tend to be efficient in explaining certain empirical phenomena, as for instance, the celebrated equity premium puzzle (see for example $[1,2,6]$ ). A further beneficial feature of the habit preferences is based on remarkably appealing grounds that are intuitively reasonable from both an economic and psychological viewpoint. Individuals who consume portions of their wealth over time are expected to develop habits which will have a

R. Muraviev ( $\varangle)$

Department of Mathematics and RiskLab, ETH Zurich, Rämistrasse 101, 8092 Zurich, Switzerland

e-mail: roman.muraviev@ math.ethz.ch 
decisive impact on their subsequent consumption behavior. In particular, the relative desire to consume may be increased if one has become accustomed to high levels of consumption. The paradigm of habit-forming utility functions captures the above observations by incorporating the impact of past consumption patterns on the individuals' current and future policy resolutions. A broad range of works are devoted to the study of habit-formation optimization problems in various contexts and applications [4,5,8-10,13,16,21].

In the current article, we provide a detailed characterization of the optimal consumption stream for the additive habit-forming utility maximization problem, in a framework of general discrete-time incomplete markets and random endowments. This characterization allows us to derive the monotonicity and concavity of the optimal consumption as a function of wealth, for several important classes of incomplete markets and preferences.

Utility maximization problems in incomplete markets with the presence of random endowments are known to be particularly challenging to handle in both discrete and continuous time, even for standard, time-separable preferences. Since the pioneering work of Merton [26], this problem has attracted the attention of many authors (see, e.g., [17, 18, 20,30,25]). However, even though existence and uniqueness of the optimal consumption has been established in a very general setting (see e.g., [21], and the references therein), very little is known about the precise structure of the optimal consumption. As emphasized in the survey paper of Zariphopoulou [32], further efforts are to be made in order to capture the qualitative structure of the optimal consumption policy.

The presence of habits makes the problem more complicated due to the natural timeinseparability of the underlying preferences. In the present article, we study an individual's life-cycle consumption problem in a fairly general discrete-time setting. A decision maker is represented by a stream of (unspanned) random endowments and an additive habitforming utility function. Agents are imposed to consume in an addictive fashion: Since the utility function is defined on the positive half-line, consumption can never fall below the benchmark level. The specification of habits in our model involves a combination of an internal-external additive mechanism. Namely, the index indicating the standard of living of an individual is a weighted average of individual's past consumption plus some exogenous (stochastic) factor. The agent is aiming to maximize the preference functional by trading in an arbitrary incomplete financial market. In the above framework, we provide a novel characterization of the optimal consumption stream by exploiting the concept of an aggregate state price density, introduced by Malamud and Trubowitz [25]. We analyze some important special cases and study particular qualitative properties of the consumption stream.

To the best of our knowledge, this article is the first one to study the habit-forming utility maximization problem in the general framework of incomplete markets and random endowments. Furthermore, unlike the traditional dynamic programming approach, our methodology allows us to explicitly characterize the optimal consumption stream for such a general setting. The approach introduced in this work can be employed in a future research dealing with habits in discrete-time models. Finally, our results allow us to address several important economic questions such as the monotonicity and concavity of the optimal consumption. In the standard case of time-separable preferences, these properties have been investigated by Carroll and Kimball [3] and Malamud and Trubowitz [25], providing a theoretical foundation for the conjecture of Keynes [23] that the marginal propensity to consume is diminishing. Our new techniques allows us to extend these results to the case of habit-forming preferences. This extension is by no means trivial as the nature of first order conditions is significantly more complicated due to the time-inseparability of the habit-forming preferences.

We now outline the contents of this article. Section 2 deals with preliminaries. In Sect. 2.1 we introduce the model of an incomplete financial market. The aggregate state price density 
and other related concepts are introduced in Sect. 2.2. In Sect. 2.3, we formulate the habitforming utility maximization problem and provide a first-phase solution (Theorem 2.3) in terms of the aggregate state price density. Section 3 is concerned with a delicate analysis of the utility maximization problem in various well-known models of financial markets and habitpreferences. This section can serve as an introduction to the more complex ideas appearing later in Sects. 4 and 5. Section 3.1 provides a solution to the optimization problem with preferences represented by a habit-forming power (constant relative risk aversion) utility, arbitrary incomplete markets and deterministic endowments. In Sect. 3.2, we consider a habit-forming exponential utility maximization problem in the case where agents can only trade one period riskless bonds. Finally, in Sect. 3.3 we solve the utility maximization problem in complete markets and arbitrary habit-forming preferences. Section 4 is devoted to the study of monotonicity, and to the establishment of an extended characterization of the consumption for a large class of incomplete markets. First, we explain in Sect. 4.1 why standard dynamic programming methods cannot be directly applied to this monotonicity problem. Then, in Sect. 4.2, the main result of Sect. 4 (Theorem 4.1) is presented: The monotonicity feature and an explicit recursive scheme determining the optimal consumption is established for arbitrary incomplete markets with a deterministic interest rate, and for idiosyncratically incomplete markets (see Definition 4.1), in the setting of general additive habit-forming preferences. In Sect. 5, we study the concavity of the optimal consumption as a function of wealth. That is, we show that the richer an individual is, the smaller is the portion of the wealth consumed by him, approving the hypothesis of Keynes [23]. Sections 5.1 and 5.2 are devoted to some surprising counter-examples illustrating that concavity can only be anticipated for time-consistent power utility functions. Finally, in Sect. 5.3, we formulate the main result of Sect. 5 (Theorem 5.2), showing the concavity property for idiosyncratically incomplete markets, and incomplete markets of type $\mathcal{C}$ with a deterministic interest rate (see Definition 4.2).

\section{Setting and preliminary results}

The uncertainty in our model is captured by a finite probability space $(\Omega, \mathcal{G}, P)$ and a filtration $\mathcal{G}_{0}:=\{\phi, \Omega\} \subseteq \mathcal{G}_{1} \subseteq \cdots \subseteq \mathcal{G}_{T}:=\mathcal{G}$, where each sigma-field $\mathcal{G}_{k}$ corresponds to the information revealed up to the period $k$. In the current article, adaptedness of stochastic processes is always meant with respect to $\mathcal{G}:=\left(\mathcal{G}_{k}\right)_{k=0, \ldots, T}$, unless otherwise is stated. For each $k, L^{2}\left(\mathcal{G}_{k}\right)$ denotes the (finite-dimensional) space of all $\mathcal{G}_{k}$-measurable random variables endowed with the inner product $\langle X, Y\rangle:=E[X \cdot Y]$, for $X, Y \in L^{2}\left(\mathcal{G}_{k}\right)$. Each sigma-algebra $\mathcal{G}_{k}$ is generated by a certain partition of $\Omega$, that is,

$$
\mathcal{G}_{k}=\sigma\left(B_{1}^{(k)}, \ldots, B_{N_{k}}^{(k)}\right),
$$

where $B_{1}^{(k)}, \ldots, B_{N_{k}}^{(k)}$ are disjoint subsets of $\Omega, \bigcup_{i=1}^{N_{k}} B_{i}^{(k)}=\Omega$ and $N_{k}=\operatorname{dim}\left(L^{2}\left(\mathcal{G}_{k}\right)\right)$. Furthermore, each partition is finer than the previous one, i.e., $B_{j}^{(k)}=\bigcup_{i \in I_{j}^{(k)}} B_{i}^{(k+1)}$, for $k=0, \ldots, T-1$, where $I_{j}^{(k)} \subseteq\left\{1, \ldots, N_{k+1}\right\}$ are disjoint subsets, for $j=1, \ldots, N_{k}$. Note that a random variable $X$ is $\mathcal{G}_{k}$ measurable if and only if $X$ is constant on each set $B_{i}^{(k)}$, for $i=1, \ldots, N_{k}$. For each $X \in L^{2}\left(\mathcal{G}_{k}\right)$, we consider the associated multiplication operator $T_{X}: L^{2}\left(\mathcal{G}_{T}\right) \rightarrow L^{2}\left(\mathcal{G}_{T}\right)$ defined by $T_{X}(Y)=X Y$. In the standard basis of $L^{2}\left(\mathcal{G}_{T}\right)$, the operator $T_{X}$ is a diagonal matrix with entries equal to $\left.X\right|_{B_{1}^{(k)}}, \ldots,\left.X\right|_{B_{N_{k}}^{(k)}}$ that appear according to a certain multiplicity. We will not distinguish between $X$ and $T_{X}$ in the forthcoming sections. Given a function $f(X, Y): \mathcal{O} \times V \rightarrow L^{2}\left(\mathcal{G}_{T}\right)$, where $\mathcal{O} \subseteq L^{2}\left(\mathcal{G}_{k}\right)$ is an 
open set (in the $L^{2}\left(\mathcal{G}_{k}\right)$-topology), for $k=0, \ldots, T$, and $V$ is some non-empty subset of $L^{2}\left(\mathcal{G}_{0}\right) \times \cdots \times L^{2}\left(\mathcal{G}_{T}\right)$, we denote by

$$
\left.\frac{\partial f}{\partial X}\right|_{\left(X_{0}, Z_{0}\right)} Y_{0}=\lim _{\varepsilon \rightarrow 0} \frac{f\left(X_{0}+\varepsilon Y_{0}, Z_{0}\right)-f\left(X_{0}, Z_{0}\right)}{\varepsilon},
$$

the directional derivative (Gâteaux differential) of $f$ at the point $\left(X_{0}, Z_{0}\right) \in \mathcal{O} \times V$, applied on the vector $Y_{0} \in L^{2}\left(\mathcal{G}_{k}\right)$. In many cases, the operator $\frac{\partial f}{\partial X}$ will be a multiplication operator, and thus $\frac{\partial f}{\partial X}$ will be treated as a random variable. Derivatives of higher order are defined in a similar manner.

\subsection{The financial market}

We adopt a standard discrete time model in the spirit of Chapter 2 in Duffie [11]. The financial market consists of $N$ risky securities and one riskless bond. There are $T+1$ periods: $0, \ldots, T$. At each date $k=0, \ldots, T-1$, each security $i=1, \ldots, N$ is available for trading (selling or buying) at the price $S_{k}^{i}$ and pays a dividend $d_{k+1}^{i}$ in the next period $k+1$. A riskless bond bought in the period $k=0, \ldots, T-1$, pays an interest rate $r_{k+1}$ in the next period $k+1$. The price process is an $N+1$ dimensional positive adapted process given by $S_{k}=\left(1, S_{k}^{1}, \ldots, S_{k}^{N}\right), k=0, \ldots, T-1$. We assume that no trading is executed in the last period $T$, and incorporate this assumption by imposing $S_{T}=(0, \ldots, 0) \in R^{N+1}$. The dividend processes is an $N+1$-dimensional adapted process denoted by $d_{k}=\left(r_{k}, d_{k}^{1}, \ldots, d_{k}^{N}\right)$, for $k=1, \ldots, T-1$ and $d_{T}=\left(1+r_{T}, d_{T}^{1}, \ldots, d_{T}^{N}\right)$. The interest rate $\left(r_{k}\right)_{k=1, \ldots, T}$ is assumed to be a non-negative predictable process. We emphasize that $\left(d_{k}\right)_{k=1, \ldots, T-1}$ and $d_{T}$ differ in the first coordinate due to the assumption that $S_{T}$ vanishes. Moreover, the first coordinate in $\left(d_{k}\right)_{k=1, \ldots, T}$ to $\left(d_{k}\right)_{k=1, \ldots, T}$ and $\left(S_{k}\right)_{k=0, \ldots, T-1}$ is reserved for the riskless bond. A trading strategy, or portfolio, is an $N+1$ dimensional adapted process $\pi_{k}=\left(\phi_{k}, \pi_{k}^{1}, \ldots, \pi_{k}^{N}\right), k=$ $0, \ldots, T$. Here, $\phi_{k}$ and $\pi_{k}^{i}$ represent the respective shares of the riskless bond and security $i$, held during the period of time $[k, k+1)$. We set further $\pi_{-1}=(0, \ldots, 0) \in R^{N+1}$ and $\pi_{T}=(0, \ldots, 0) \in R^{N+1}$. One notes that the standard assumption regarding the predictability of the portfolio is omitted due to a shift in the index of the price process. For a given trading strategy $\pi$, the associated investment process $I^{\pi}:=\left(I_{k}^{\pi}\right)_{k=0, \ldots, T-1}$ and the associated financial wealth processes $W^{\pi}:=\left(W_{k}^{\pi}\right)_{k=1, \ldots, T}$ are defined respectively by

$$
I_{k}^{\pi}:=\phi_{k}+\sum_{i=1}^{N} \pi_{k}^{i} S_{k}^{i}=\pi_{k} \cdot S_{k}
$$

and

$$
W_{k}^{\pi}:=\phi_{k-1}\left(1+r_{k}\right)+\sum_{i=1}^{N} \pi_{k-1}^{i}\left(S_{k}^{i}+d_{k}^{i}\right)=\pi_{k-1} \cdot\left(S_{k}+d_{k}\right),
$$

where $\cdot$ denotes the standard inner product in $R^{N+1}$. A state price density $(S P D)$ is an adapted process $\left(R_{k}\right)_{k=0, \ldots, T}$ that satisfies

$$
R_{k} S_{k}^{i}=E\left[R_{k+1}\left(S_{k+1}^{i}+d_{k+1}^{i}\right) \mid G_{k}\right]
$$

and

$$
R_{k}=E\left[R_{k+1}\left(1+r_{k+1}\right) \mid G_{k}\right],
$$

for all $i=1, \ldots, N$ and $k=0, \ldots, T-1$. 
Remark 2.1 (i) In our setting, a SPD is allowed to take negative values.

(ii) Given an investment process $\left(I_{k}^{\pi}\right)_{k=0, \ldots, T-1}$, a wealth process $\left(W_{k}^{\pi}\right)_{k=0, \ldots, T}$ and an arbitrary $\operatorname{SPD}\left(R_{k}\right)_{k=0, \ldots, T}$, the following relation

$$
R_{k} I_{k}^{\pi}=E\left[R_{k+1} W_{k+1}^{\pi} \mid \mathcal{G}_{k}\right]
$$

holds for all $k=0, \ldots, T-1$.

The following is assumed throughout the whole article.

Assumption 1 There is no arbitrage (NA) in the market. That is, if, $W_{k}^{\pi}-I_{k}^{\pi} \geq 0, P$-a.s for all $k=0, \ldots, T$ and some portfolio $\pi$, then, $W_{k}^{\pi}-I_{k}^{\pi}=0, P-$ a.s for all $k=0, \ldots, T$.

We recall the standard classification of financial markets.

Definition 2.1 A market is complete if every adapted process $\left(Y_{k}\right)_{k=1, \ldots, T}$ is replicable, i.e., there exists a trading strategy $\pi$ such that $Y_{k}=I_{k}^{\pi}-W_{k}^{\pi}$ for all $k=1, \ldots, T$. Otherwise, the market is called incomplete.

Finally, we state the following classical result in a discrete time setting.

Theorem 2.1 The NA condition is equivalent to the existence of a positive SPD. A NA market is complete if and only if there exists a unique (up to a multiplication by a constant) positive $S P D$.

Proof of Theorem 2.1 See the proof of Theorem 3.3 in Dallang et al. [7], and the proof of Corollary 4.1 in Taqqu and Willinger [31].

2.2 The aggregate state price density

The current subsection is devoted to the introduction of certain important notions that will be crucial for carrying out our analysis. We start with the following definition.

Definition 2.2 (i) The financial wealth space, or payoff space at period $k$ is given by

$$
\mathcal{L}_{k}=\left\{W_{k}^{\pi}=\left(S_{k}+d_{k}\right) \pi_{k-1} \mid \pi_{k-1} \in L^{2}\left(\mathcal{G}_{k-1}\right)\right\},
$$

for all $k=1, \ldots, T$, and $\mathcal{L}_{0}=\{0\}$.

(ii) We denote by

$$
P_{\mathcal{L}}^{k}: L^{2}\left(\mathcal{G}_{T}\right) \rightarrow \mathcal{L}_{k}
$$

the orthogonal projection of the space $L^{2}\left(\mathcal{G}_{T}\right)$ onto the payoff space $\mathcal{L}_{k}$, for all $k=$ $0, \ldots, T$.

Remark 2.2 (i) Since riskless bonds are available for trading at each period, it follows that $L^{2}\left(\mathcal{G}_{k-1}\right) \subseteq \mathcal{L}_{k} \subseteq L^{2}\left(\mathcal{G}_{k}\right)$, for all $k=1, \ldots, T$.

(ii) In contrast to conditional expectations, the orthogonal projection $P_{\mathcal{L}}^{k}$ is not necessarily positivity preserving (i.e., not mapping non-negative random variables to nonnegative ones). In fact, $P_{\mathcal{L}}^{k}$ is positivity preserving if and only if $\mathcal{L}_{k}=L^{2}\left(\mathcal{H}_{k}\right)$ for some sigma-algebra $\mathcal{G}_{k-1} \subseteq \mathcal{H}_{k} \subseteq \mathcal{G}_{k}$ (See Theorem 3.2 in [25]). In the latter case, the market is called market of type $\mathcal{C}$ (see Sects. 4.2 and 5.3 for certain results involving this type of markets).

The following properties will be used extensively in the sequel. 
Lemma 2.1 (i) For every $X, Y \in L^{2}\left(\mathcal{G}_{T}\right)$ and $k=1, \ldots, T$, we have

$$
E\left[P_{\mathcal{L}}^{k}[X] Y\right]=E\left[X P_{\mathcal{L}}^{k}[Y]\right] .
$$

(ii) For every $k=1, \ldots, T, Y \in L^{2}\left(\mathcal{G}_{T}\right)$ and $X \in L^{2}\left(\mathcal{G}_{k-1}\right)$, we have

$$
P_{\mathcal{L}}^{k}[X Y]=X P_{\mathcal{L}}^{k}[Y] .
$$

(iii) For all $X, Y \in L^{2}\left(\mathcal{G}_{T}\right), k=0, \ldots, T-1$ and $m>k$, we have

$$
E\left[P_{\mathcal{L}}^{m}[X] Y \mid \mathcal{G}_{k}\right]=E\left[X P_{\mathcal{L}}^{m}[Y] \mid \mathcal{G}_{k}\right] .
$$

(iv) If $P_{\mathcal{L}}^{k}[X V]=0$, for some positive random variable $X \in L^{2}\left(G_{T}\right)$ and some $V \in \mathcal{L}_{k}$, then $V=0$.

Proof of Lemma $2.1 \quad$ (i) The assertion follows from the fact that $P_{\mathcal{L}}^{k}$ is a self-adjoint operator.

(ii) First, note that $X P_{\mathcal{L}}^{k}[Y] \in \mathcal{L}_{k}$ by definition. Therefore, the statement becomes $P_{\mathcal{L}}^{k}\left[X Y-X P_{\mathcal{L}}^{k}[Y]\right]=0$, or equivalently, $E\left[\left(X Y-X P_{\mathcal{L}}^{k}[Y]\right) Z\right]=0$, for all $Z \in \mathcal{L}_{k}$. Since $X Z \in \mathcal{L}_{k}$, part (i) implies that $E\left[X Z P_{\mathcal{L}}^{k}[Y]\right]=E[X Y Z]$. This completes the proof of (ii).

(iii) We need to prove that $E\left[P_{\mathcal{L}}^{m}[X] Y Z\right]=E\left[P_{\mathcal{L}}^{m}[Y] X Z\right]$, for all $Z \in L^{2}\left(\mathcal{G}_{k}\right)$. It follows by (ii) that $Z P_{\mathcal{L}}^{m}[X]=P_{\mathcal{L}}^{m}[Z X]$. Therefore, by combining this with (i) we obtain that $E\left[P_{\mathcal{L}}^{m}[X] Y Z\right]=E\left[P_{\mathcal{L}}^{m}[X Z] Y\right]=E\left[P_{\mathcal{L}}^{m}[Y] X Z\right]$, as required.

(iv) Note that by (i) we have $0=E\left[P_{\mathcal{L}}^{k}[X V] V\right]=E\left[X V^{2}\right]$. Since $X>0$, we conclude that $V=0$.

The concept of aggregate SPD is a fundamental tool in the present work.

Theorem 2.2 There exists a unique SPD $\left(M_{k}\right)_{k=0, \ldots, T}$ such that $M_{k} \in \mathcal{L}_{k}$, for all $k=$ $1, \ldots, T$. Moreover,

$$
M_{k}=\prod_{l=1}^{k} P_{\mathcal{L}}^{l}\left[\frac{R_{l}}{R_{l-1}}\right],
$$

for all $k=1, \ldots, T$, where $\left(R_{k}\right)_{k=0, \ldots, T}$ is an arbitrary positive SPD. In particular, $\left(M_{k}\right)_{k=0, \ldots, T}$ is independent of the choice of $\left(R_{k}\right)_{k=0, \ldots, T}$. The process $\left(M_{k}\right)_{k=0, \ldots, T}$ is called the aggregate SPD.

Proof of Theorem 2.2 See the proof of Lemma 2.5 in Malamud and Trubowitz [25].

Note that the aggregate SPD is not necessarily a positive process. For simplicity, we will consider only financial markets that satisfy the following convention.

Assumption 2 The aggregate SPD does not vanish, i.e., $M_{k} \neq 0, P-$ a.s, for all $k=$ $1, \ldots, T$.

\subsection{Utility maximization with additive habits}

The decision maker in our model is characterized by an endowment stream $\epsilon:=\left(\epsilon_{k}\right)_{k=0, \ldots, T}$ which is assumed to be a non-negative adapted process, and a habit-forming utility function 
$U$. The individual's objective is to maximize her utility function from consumption by selecting a suitable investment policy. Given a trading strategy $\pi$, the corresponding consumption process labeled by $c^{\pi}:=\left(c_{k}^{\pi}\right)_{k=0, \ldots, T}$, is defined by

$$
c_{k}=c_{k}^{\pi}:=\epsilon_{k}+W_{k}^{\pi}-I_{k}^{\pi} .
$$

The preference functional of the agent is given by

$$
U\left(c_{0}, \ldots, c_{T}\right):=\sum_{k=0}^{T} E\left[u_{k}\left(c_{k}-\sum_{l=0}^{k-1} \beta_{l}^{(k)} c_{l}-h_{k}\right)\right],
$$

where $\beta:=\left(\beta_{l}^{(k)}\right)_{l=0, \ldots, k-1}$, for $k=1, \ldots, T$, are non-negative constants that represent the strength of the habit formation affect on the individual, and $\left(h_{k}\right)_{k=0, \ldots, T}$ is a non-negative adapted process that might be interpreted as a quantity which measures habits of exogenous type, or as a mending factor that appears due to a noisy estimation of the consumption's past history. For notational convenience, we set $\beta_{-1}^{(0)}=h_{0}=0$ and $\beta_{k}^{(k)}=1$, for $k=$ $1, \ldots, T$. We assume that each function $u_{k}:[0, \infty) \rightarrow R$ is a strictly increasing, concave, $C^{2}$-smooth and satisfies the Inada conditions: $u_{k}^{\prime}(0):=\lim _{x \rightarrow 0} u_{k}^{\prime}(x)=\infty$, and $u_{k}^{\prime}(\infty):=$ $\lim _{x \rightarrow \infty} u_{k}^{\prime}(x)=0$. Note that the latter convention implies that feasible consumption streams are non-negative and that individuals are addictive, that is, $c_{k} \geq \sum_{l=0}^{k-1} \beta_{l}^{(k)} c_{l}+h_{k} \geq 0$, for all $k=0, \ldots, T$. For each consumption plan $\left(c_{k}\right)_{k=0, \ldots, T}$ we define the associated perturbed consumption process $(c(k, \beta, h))_{k=0, \ldots, T}$ by

$$
c(k, \beta, h):=c_{k}-\sum_{l=0}^{k-1} \beta_{l}^{(k)} c_{l}-h_{k} .
$$

Note that the utility functions in the current setting are not restricted by the assumption that $u_{k}(x)=e^{-\rho k} u(x)$, for all $k=0, \ldots, T$, where $u(x)$ is some function satisfying the Inada conditions. This leads to time-inconsistency, as for instance in Ekeland and Lazrak [14], and Karp [22]. The following subset of $L^{2}\left(\mathcal{G}_{0}\right) \times L^{2}\left(\mathcal{G}_{1}\right) \times \cdots \times L^{2}\left(\mathcal{G}_{T}\right)$ will be referred to as the set of budget constraints

$$
\begin{aligned}
B \quad & (\epsilon, \beta, h):= \\
& \left\{\left(c_{0}, \ldots, c_{T}\right) \mid c_{k}=c_{k}^{\pi} \text { for some portfolio } \pi \text { and } c(k, \beta, h) \geq 0 \text { for all } k=0, \ldots, T\right\} .
\end{aligned}
$$

We assume throughout the article that the set $B(\epsilon, \beta, h)$ has a non-empty interior. The utility maximization problem of the agent is

$$
\sup _{\left(c_{0}, \ldots, c_{T}\right) \in B(\epsilon, \beta, h)} U\left(c_{0}, \ldots, c_{T}\right) .
$$

The following proposition provides a convenient parametrization of the set of budget constraints.

Lemma 2.2 Let $\left(c_{k}\right)_{k=0, \ldots, T}$ be an adapted process that satisfies the condition $c(k, \beta, h) \geq$ 0 , for all $k=0, \ldots, T$. Then, $\left(c_{k}\right)_{k=0, \ldots, T}$ is a feasible consumption stream, i.e., $\left(c_{0}, \ldots, c_{T}\right) \in B(\epsilon, \beta, h)$, if and only if there exists a unique wealth process $\left(W_{k}\right)_{k=0, \ldots, T}$, i.e., $W_{k} \in \mathcal{L}_{k}$ for all $k=0, \ldots, T$ and $W_{T+1}=0$, such that

$$
c_{k}=\epsilon_{k}+W_{k}-E\left[\frac{M_{k+1}}{M_{k}} W_{k+1} \mid \mathcal{G}_{k}\right],
$$


for all $k=0, \ldots, T$. Furthermore, if $\pi$ is some trading strategy such that $c_{k}=c_{k}^{\pi}$ for all $k=0, \ldots, T$, then $W_{k}=W_{k}^{\pi}$ and $E\left[\frac{M_{k+1}}{M_{k}} W_{k+1} \mid \mathcal{G}_{k}\right]=I_{k}^{\pi}$, for all $k=0, \ldots, T$. The wealth process $\left(W_{k}\right)_{k=0, \ldots, T}$ is uniquely determined by

$$
W_{k}=\sum_{l=k}^{T} E\left[\frac{M_{l}}{M_{k}}\left(c_{l}-\epsilon_{l}\right) \mid \mathcal{G}_{k}\right]
$$

for all $k=1, \ldots, T$.

This lemma is analogous to Lemma 2.7 in Malamud and Trubowitz [25]. We present its proof for the reader's convenience.

Proof of Lemma 2.2 The first part of the statement follows by (1). Let us now show that (6) holds by backward induction. The case $k=T$ follows from (5). Assume that (6) holds for all $k>m$, and let us show it for $k=m$. By combining Eq. 5 with the induction assumption, we get that $W_{m}=c_{m}-\epsilon_{m}+E\left[\frac{M_{m+1}}{M_{m}} W_{m+1} \mid \mathcal{G}_{m}\right]=c_{m}-\epsilon_{m}+\sum_{l=m+1}^{T} E\left[\frac{M_{m+1}}{M_{m}} E\left[\frac{M_{l}}{M_{m+1}}\left(c_{l}-\right.\right.\right.$ $\left.\left.\left.\epsilon_{l}\right) \mid \mathcal{G}_{m+1}\right] \mid \mathcal{G}_{m}\right]=\sum_{l=m}^{T} E\left[\frac{M_{l}}{M_{m}}\left(c_{l}-\epsilon_{l}\right) \mid \mathcal{G}_{m}\right]$. This accomplishes the proof.

The next statement establishes a first step in the characterization of the optimal consumption stream in the setting of general incomplete markets.

Theorem 2.3 There exists a unique solution to the utility maximization problem (4). The optimal consumption stream $\left(c_{k}^{*}\right)_{k=0, \ldots, T}$ and the corresponding financial wealth process $\left(W_{k}^{*}\right)_{k=0, \ldots, T}$ are uniquely determined as the solutions of the following system of equations:

$$
P_{\mathcal{L}}^{k}\left[\frac{R_{k}\left(c_{0}, \ldots, c_{T}\right)}{R_{k-1}\left(c_{0}, \ldots, c_{T}\right)}\right]=\frac{M_{k}}{M_{k-1}},
$$

for $k=1, \ldots, T$, where

$$
R_{k}\left(c_{0}, \ldots, c_{T}\right):=u_{k}^{\prime}(c(k, \beta, h))-\sum_{m=k+1}^{T} \beta_{k}^{(m)} E\left[u_{m}^{\prime}(c(m, \beta, h)) \mid \mathcal{G}_{k}\right],
$$

$(c(k, \beta, h))_{k=0, \ldots, T}$ is defined in (3),

$$
c_{k}=\epsilon_{k}+W_{k}-E\left[\frac{M_{k+1}}{M_{k}} W_{k+1} \mid \mathcal{G}_{k}\right],
$$

and $W_{k} \in \mathcal{L}_{k}$, for all $k=0, \ldots, T$. The process $\left(R_{k}\left(c_{0}^{*}, \ldots, c_{T}^{*}\right)\right)_{k=0, \ldots, T}$ is a positive SPD.

Proof of Theorem 2.3 The compactness of $B(\epsilon, \beta, H)$ (which follows by the NA assumption) combined with the continuity and the strict concavity of the function $U\left(c_{0}, \ldots, c_{T}\right)$ implies existence and uniqueness. Set $c^{*}\left(k, \beta, h_{k}\right):=c_{k}^{*}-\sum_{l=0}^{k-1} \beta_{l}^{(k)} c_{l}^{*}-h_{k}$ and observe that the Inada condition $u_{k}^{\prime}(0)=\infty$ implies that $c^{*}\left(k, \beta, h_{k}\right)>0, P-$ a.s, for all $k=0, \ldots, T$. We turn to showing that $\left(c_{k}^{*}\right)_{k=0, \ldots, T}$ is determined as the unique solution of the system of Eqs. 7 and 8. Note that Lemma 2.2 implies that Eq. 8 can replace the budget constraint $\left(c_{0}^{*}, \ldots, c_{T}^{*}\right) \in B(\epsilon, \beta, h)$. Next, due to the fact that $\left(c_{0}^{*}, \ldots, c_{T}^{*}\right) \in \operatorname{int}(B(\epsilon, \beta, h))$ and that a local maximum of a strict concave function is a global maximum, it follows that $\left(c_{0}^{*}, \ldots, c_{T}^{*}\right)$ is determined as the unique solution of the corresponding first order conditions, which are given by

$$
R_{k}\left(c_{0}, \ldots, c_{T}\right) S_{k}^{i}=E\left[R_{k+1}\left(c_{0}, \ldots, c_{T}\right)\left(S_{k+1}^{i}+d_{k+1}^{i}\right) \mid \mathcal{G}_{k}\right],
$$


and

$$
R_{k}\left(c_{0}, \ldots, c_{T}\right)=E\left[R_{k+1}\left(c_{0}, \ldots, c_{T}\right)\left(1+r_{k+1}\right) \mid \mathcal{G}_{k}\right],
$$

for all $i=0, \ldots, N$ and $k=0, \ldots, T-1$. Thereby, we conclude that the process $\left(R_{k}\left(c_{0}^{*}, \ldots, c_{T}^{*}\right)\right)_{k=0, \ldots, T}$ is a SPD. Now, observe that $R_{T}\left(c_{0}^{*}, \ldots, c_{T}^{*}\right)=u_{T}^{\prime}\left(c^{*}(T, \beta, h)\right)>$ 0 by definition, and hence the positivity of the process $\left(R_{k}\left(c_{0}^{*}, \ldots, c_{T}^{*}\right)\right)_{k=0, \ldots, T}$ follows by the above first order conditions and the fact that the processes $\left(S_{k}\right)_{k=0, \ldots, T},\left(d_{k}\right)_{k=1, \ldots, T}$ and $\left(r_{k}\right)_{k=1, \ldots, T}$ are positive. Finally, the proof is accomplished by applying Theorem 2.2 on $\left(R_{k}\left(c_{0}^{*}, \ldots, c_{T}^{*}\right)\right)_{k=0, \ldots, T}$ and combining this with the convention that the aggregate SPD $\left(M_{k}\right)_{k=0, \ldots, T}$ does not vanish.

For the sake of convenience, the following notation will be used in the sequel.

Definition 2.3 The process $\left(\tilde{M}_{k}\right)_{k=0, \ldots, T}$, given by

$$
\widetilde{M}_{k}=M_{k}+\sum_{l=k+1}^{T} \sum_{j=1}^{l-k} \sum_{k \leq s_{j}<\cdots<s_{1}<l} \beta_{s_{1}}^{(l)} \beta_{s_{2}}^{\left(s_{1}\right)} \cdots \beta_{k}^{\left(s_{j}\right)} E\left[M_{l} \mid G_{k}\right],
$$

is referred as the aggregate perturbed SPD.

\section{Optimal consumption for some special classes of financial markets and preferences}

The system of first order Eq. 7 appearing in Theorem 2.3 is highly non-linear and closed form solutions can only be obtained in some special settings. In the current section, we study several such cases. As it will be demonstrated in the subsequent three subsections, these closed form solutions provide a detailed description of the structure of the optimal consumption stream in the presence of habits, and allow illustrating certain important characteristics.

\subsection{Power utility with no random endowment}

Consider an agent represented by a power utility $u_{k}(x)=e^{-\rho k} \frac{x^{1-\gamma}}{1-\gamma}$, for $k=0, \ldots, T$. Here, $\gamma$ and $\rho$ stand for the degree of risk aversion and impatience respectively. Assume that the agent receives only an initial endowment, that is, $\epsilon_{0} \geq 0$, and $\epsilon_{k}=0$, for all $k=1, \ldots, T$. Assume further that no exogenous habits are involved, namely, $h_{k}=0$, for all $k=1, \ldots, T$. We denote by $c_{k}\left(\epsilon_{0}\right), W_{k}\left(\epsilon_{0}\right)$ and $I_{k}\left(\epsilon_{0}\right)$ the optimal consumption, wealth and investment processes respectively, viewed as functions of the initial endowment $\epsilon_{0}$. The solution to the corresponding utility maximization problem is given explicitly in the following statement.

Theorem 3.1 Under the above assumptions, the optimal consumption and investment policies are linear increasing functions of the wealth, that is,

$$
c_{k}\left(\epsilon_{0}\right)=A_{k} W_{k}\left(\epsilon_{0}\right) ; \quad c_{0}\left(\epsilon_{0}\right)=A_{0} \epsilon_{0},
$$

and

$$
I_{k}\left(\epsilon_{0}\right)=\left(1-A_{k}\right) W_{k}\left(\epsilon_{0}\right) ; \quad I_{0}\left(\epsilon_{0}\right)=\left(1-A_{0}\right) \epsilon_{0},
$$

for all $k=1, \ldots, T$, where $\left(A_{k}\right)_{k=0, \ldots, T}$ is an adapted processes taking values in the interval $(0,1]$, which is given by

$$
A_{k}=\frac{c_{k}(1)}{c_{k}(1)+E\left[\frac{M_{k+1}}{M_{k}} W_{k+1}(1) \mid \mathcal{G}_{k}\right]} .
$$


Moreover, the corresponding wealth process $\left(W_{k}\left(\epsilon_{0}\right)\right)_{k=1, \ldots, T}$ satisfies

$$
W_{k+1}\left(\epsilon_{0}\right)=\frac{W_{k+1}(1)}{c_{k}(1)} c_{k}\left(\epsilon_{0}\right)
$$

for all $k=0, \ldots, T-1$.

Proof of Theorem 3.1 First, observe that the first order conditions (7) in Theorem 2.3 imply that $c_{k}\left(\epsilon_{0}\right)=c_{k}(1) \epsilon_{0}$ and $W_{k}\left(\epsilon_{0}\right)=W_{k}(1) \epsilon_{0}$, for all $k=0, \ldots, T$. This implies that $W_{k+1}\left(\epsilon_{0}\right)=\frac{W_{k+1}(1)}{c_{k}(1)} c_{k}\left(\epsilon_{0}\right)$, for all $k=0, \ldots, T-1$, and by plugging this into Eq. 8, we conclude the validity of (10). Next, notice that relation (11) holds due to Lemma 2.2. We claim now that $E\left[\frac{M_{k+1}}{M_{k}} W_{k+1}(1) \mid \mathcal{G}_{k}\right]>0, P-$ a.s., for all $k=0, \ldots, T-1$. This is unclear a-priori since the aggregate $\operatorname{SPD}\left(M_{k}\right)_{k=0, \ldots, T}$ is not necessarily positive. To this end, set $\epsilon_{0}=1$ and note that by (8), we have $W_{T}(1)=c_{T}(1)>0$. Therefore, by item (ii) in Remark (2.1), we get

$$
E\left[\frac{M_{T}}{M_{T-1}} W_{T}(1) \mid \mathcal{G}_{T-1}\right]=E\left[\frac{R_{T}}{R_{T-1}} W_{T}(1) \mid \mathcal{G}_{T-1}\right]>0,
$$

where $\left(R_{k}\right)_{k=0, \ldots, T}$ is an arbitrary positive SPD. Next, note that (2.2) implies that $W_{T-1}(1)=c_{T-1}(1)+E\left[\frac{M_{T}}{M_{T-1}} W_{T}(1) \mid \mathcal{G}_{T-1}\right]>0$. Hence, as above, we conclude that $E\left[\frac{M_{T-1}}{M_{T-2}} W_{T-1}(1) \mid \mathcal{G}_{T-2}\right]=E\left[\frac{R_{T-1}}{R_{T-2}} W_{T-1}(1) \mid \mathcal{G}_{T-2}\right]>0$. The rest of the claim can be proved analogously by using backward induction.

\subsection{Exponential utility and one period risk free bonds}

Consider a market which consists only of one period risk free bonds represented by an interest rate process $\left(r_{k}\right)_{k=1, \ldots, T}$. One checks that $\mathcal{L}_{k}=L^{2}\left(\mathcal{G}_{k-1}\right)$ (in particular, this implies that $\left.P_{\mathcal{L}}^{k}[\cdot]=E\left[\cdot \mid \mathcal{G}_{k-1}\right]\right)$, and $M_{k}=\prod_{i=1}^{k}\left(1+r_{i}\right)^{-1}$, for all $k=1, \ldots, T$. The agent is represented by an exponential utility $u_{k}: R \rightarrow R_{-}, u_{k}(x)=-e^{-\gamma x}, k=0, \ldots, T$, where $\gamma>0$ stands for the degree of risk aversion. We emphasize that this specification of preferences is exceptional for the current article, since the utility function is defined on the whole real-line $R$. Note however that the Inada conditions hold in the following modified form, $u_{k}^{\prime}(-\infty)=+\infty$ and $u_{k}^{\prime}(+\infty)=0, k=0, \ldots, T$. The exogenous habits are characterized by the process $\left(h_{k}\right)_{k=0}$. The internal habits coefficients are assumed to be homogeneous and the habit consumption level is assumed to depend only on last period consumption, i.e., $\beta_{k-1}^{(k)}=\beta \geq 0$, and $\beta_{l}^{(k)}=0$, for all $k=1, \ldots, T$, and $l=0, \ldots, k-2$. The latter framework is in fact the most prevalent specification of a habit forming utility function in the discrete time literature (see e.g. $[1,15,29]$ ). The utility maximization problem in the current setting is similar to the one in (4), apart from the evident distinction that the process $\left(c_{k}\right)_{k=0, \ldots, T}$ is allowed to take negative values. Let $\left(\epsilon_{k}\right)_{k=0, \ldots, T}$ represent the (possibly random) endowment stream of the agent. The optimal consumption, investment and wealth are denoted respectively by $\left(c_{k}^{*}\right)_{k=0, \ldots, T},\left(I_{k}^{*}\right)_{k=0, \ldots, T}$ and $\left(W_{k}^{*}\right)_{k=1, \ldots, T}$.

Theorem 3.2 Under the above assumptions, the optimal consumption and investment processes are determined explicitly through the following recursive scheme,

$$
\begin{aligned}
c_{k}^{*} & =l_{k} W_{k}^{*}+m_{k} c_{k-1}^{*}+n_{k}\left(\epsilon_{k}, \ldots, \epsilon_{T}\right), \\
I_{k}^{*} & =\left(1-l_{k}\right) W_{k}^{*}-m_{k} c_{k-1}^{*}+\epsilon_{k}-n_{k}\left(\epsilon_{k}, \ldots, \epsilon_{T}\right), \\
W_{k}^{*} & =l_{k}^{\prime} c_{k-1}^{*}+m_{k}^{\prime} c_{k-2}^{*}+n_{k}^{\prime}\left(\epsilon_{k}, \ldots, \epsilon_{T}\right),
\end{aligned}
$$


for all $k=1, \ldots, T$,

$$
c_{0}^{*}=l_{0} \epsilon_{0}+m_{0} \frac{n_{0}^{\prime}}{m_{0}^{\prime}},
$$

and

$$
I_{0}^{*}=\epsilon_{0}-c_{0}^{*}
$$

where $l_{k}, m_{k}, n_{k}\left(\epsilon_{k}, \ldots, \epsilon_{T}\right), l_{k}^{\prime}, m_{k}^{\prime}$ and $n_{k}^{\prime}\left(\epsilon_{k}, \ldots, \epsilon_{T}\right)$ are given explicitly in (16), (17), (18) and (19). Furthermore, we have

$$
0<l_{k} \leq 1
$$

for all $k=0, \ldots, T$.

Proof of Theorem 3.2 As in the proof of Theorem 2.3, one checks that the optimal consumption stream is determined as the unique solution of the system of Eqs. 7 and 8 . Now, note that Eq. 8 becomes $c_{k}=\epsilon_{k}+W_{k}-\left(1+r_{k+1}\right)^{-1} W_{k+1}$, for $k=0, \ldots, T$. Thus it is evident that $l_{T}=1, m_{T}=0$ and $n_{T}\left(\epsilon_{T}\right)=\epsilon_{T}$. It is easy to check by backward induction that Eq. 7 admits the form

$$
E\left[e^{-\gamma\left(c_{k}-\beta c_{k-1}-h_{k}\right)} X_{k} \mid \mathcal{G}_{k-1}\right]=e^{-\gamma\left(c_{k-1}-\beta c_{k-2}-h_{k-1}\right)},
$$

for $k=1, \ldots, T$, where $\left(X_{k}\right)_{k=1, \ldots, T}$ is given by

$$
X_{k}=\beta+\frac{1}{\sum_{j=k}^{T} \prod_{i=j}^{T}\left(1+r_{i}\right)^{-1} \beta^{T-j}} .
$$

Finally, the above formula combined with Eq. 8 implies that

$$
\begin{aligned}
l_{k}^{\prime} & =\frac{1+\beta-m_{k}}{l_{k}} ; \quad m_{k}^{\prime}=-\frac{\beta}{l_{k}}, \\
n_{k}^{\prime}\left(\epsilon_{k}, \ldots, \epsilon_{T}\right) & =\frac{\log \left(E\left[\exp \left(\gamma\left(h_{k}-n_{k}\left(\epsilon_{k}, \ldots, \epsilon_{T}\right)\right)\right) X_{k} \mid \mathcal{G}_{k-1}\right]\right)-\gamma h_{k-1}}{\gamma l_{k}}, \\
l_{k-1} & =\frac{1}{1+\left(1+r_{k}\right)^{-1} l_{k}^{\prime}} ; \quad m_{k-1}=-\frac{\left(1+r_{k}\right)^{-1} m_{k}^{\prime}}{1+\left(1+r_{k}\right)^{-1} l_{k}^{\prime}}, \\
n_{k-1}\left(\epsilon_{k-1}, \ldots, \epsilon_{T}\right) & =\frac{\epsilon_{k-1}-\left(1+r_{k}\right)^{-1} n_{k}^{\prime}\left(\epsilon_{k}, \ldots, \epsilon_{T}\right)}{1+\left(1+r_{k}\right)^{-1} l_{k}^{\prime}},
\end{aligned}
$$

for $k=1, \ldots, T$. Finally, one checks by induction that $l_{k} \in[0,1), k=0, \ldots, T$.

\subsection{Complete markets}

By definition, the relation $L_{k}=L^{2}\left(\mathcal{G}_{k}\right)$ (and in particular, $P_{\mathcal{L}}^{k}[\cdot]=E\left[\cdot \mid \mathcal{G}_{k}\right]$ ) holds for all $k=1, \ldots, T$, in a complete market setting. Moreover, the aggregate SPD is the unique positive SPD in the market. The next statement treats the case of general habit-forming utility functions.

Theorem 3.3 The optimal consumption stream $\left(c_{k}^{*}\right)_{k=0, \ldots, T}$ is determined explicitly through the following recursive scheme,

$$
c_{k}^{*}=\sum_{l=0}^{k-1} \beta_{l}^{(k)} c_{l}^{*}+h_{k}+\left(u_{k}^{\prime}\right)^{-1}\left(u_{k-1}^{\prime}\left(c^{*}\left(k-1, \beta, h_{k-1}\right)\right) \frac{\tilde{M}_{k}}{\widetilde{M}_{k-1}}\right),
$$


for all $k=1, \ldots, T$, and

$$
\sum_{k=0}^{T} E\left[c_{k}^{*} \frac{M_{k}}{M_{k-1}}\right]=\sum_{k=0}^{T} E\left[\epsilon_{k} \frac{M_{k}}{M_{k-1}}\right],
$$

where $\left(\widetilde{M}_{k}\right)_{k=0, \ldots, T}$ is given in (9), and $\left(c^{*}(k, \beta, h)\right)_{k=0, \ldots, T}$ is defined as in (2.3).

Proof of Theorem 3.3 Since the market is complete, the budget constraint Eq. 8 are redundant for $k=1, \ldots, T$ and thus the only relevant budget constraint is Eq. 8 for $k=0$. This equation can be rewritten as (21) by identity (6) in Lemma 2.2. The first order conditions (7) can be transformed into (20) by combining the identity $P_{\mathcal{L}}^{k}[\cdot]=E\left[\cdot \mid \mathcal{G}_{k}\right], k=$ $1, \ldots, T$, with some routine, but somewhat tedious, algebraic manipulations (similarly as in Lemma 4.2).

We concentrate now on power utility functions. That is, $u_{k}(x)=e^{-\rho k} \frac{x^{1-\gamma_{k}}}{1-\gamma_{k}}$, for $k=$ $0, \ldots, T$, where $\left(\gamma_{k}\right)_{k=0, \ldots, T}$ is a sequence of non-negative numbers that represent the risk aversion of the agent, and $\rho$ indicates the impatience coefficient. In contrast to the previous two subsections, the optimal consumption stream here may demonstrate a non-linear structure. The next result illustrates the latter phenomenon, and presents an analytical solution to the associated utility maximization problem.

Theorem 3.4 (i) Under the above assumptions, we have

$$
c_{k}^{*}=\psi_{k}\left(W_{k}^{*}\right),
$$

for $k=1, \ldots, T$, and $c_{0}^{*}=\psi_{0}\left(\epsilon_{0}\right)$ where, $\psi_{k}: L^{2}\left(\mathcal{G}_{k}\right) \rightarrow L^{2}\left(\mathcal{G}_{k}\right)$ is a smooth function which is given explicitly by (28), for all $k=0, \ldots, T$. The derivative $d \psi_{k}$ is a multiplication operator such that $0<\left(d \psi_{k}\right)_{W_{k}\left(\epsilon_{0}, \ldots, \epsilon_{k-1}\right)} \leq 1$. Furthermore, we have

$$
I_{k}\left(\epsilon_{0}, \ldots, \epsilon_{k-1}\right)=\widehat{\psi}_{k}\left(W_{k}\left(\epsilon_{0}, \ldots, \epsilon_{k-1}\right)\right),
$$

for $k=1, \ldots, T$ and $I_{0}\left(\epsilon_{0}\right)=\widehat{\psi}_{k}\left(\epsilon_{0}\right)$. Here, $\widehat{\psi}_{k}: L^{2}\left(\mathcal{G}_{k}\right) \rightarrow L^{2}\left(\mathcal{G}_{k}\right)$ is given by $\widehat{\psi}_{k}(X)=\epsilon_{k}+X-\psi_{k}(X)$, for all $k=0, \ldots, T$. In particular, the optimal consumption/investment is an increasing function of the wealth.

(ii) If $\gamma_{0}=\cdots=\gamma_{T}$, then, the optimal consumption/investment stream is a linear increasing function of the wealth. More precisely,

$$
\psi_{k}(X)=\frac{\sum_{i=0}^{k} D_{k}^{(i)}}{\sum_{i=0}^{T} F_{k}^{(i)}} X+\frac{\sum_{i=0}^{k} D_{k}^{(i)}}{\sum_{i=0}^{T} F_{k}^{(i)}} \sum_{j=k}^{T} E\left[\frac{M_{j}}{M_{k}} \epsilon_{j} \mid \mathcal{G}_{k}\right]+\sum_{i=1}^{k} \alpha_{i}^{(k)} h_{i},
$$

where $D_{k}^{(i)}, \alpha_{i}^{(k)}$ and $F_{k}^{(i)}$ are given by (23), (24) and (27) respectively.

Proof of Theorem 3.4 (i) Theorem 3.3 implies that $\left(c_{k}^{*}\right)_{k=0, \ldots, T}$ satisfies the equation

$$
c_{k}^{*}-\sum_{j=0}^{k-1} \beta_{j}^{(k)} c_{j}^{*}-h_{k}=\left(c_{0}^{*}\right)^{\gamma_{0} / \gamma_{k}} e^{-\frac{\rho k}{\gamma_{k}}}\left(\tilde{M}_{k}\right)^{-1 / \gamma_{k}},
$$

for $k=1, \ldots, T$. By using routine algebraic operations, one obtains that

$$
c_{k}^{*}=\sum_{i=0}^{k} D_{k}^{(i)}\left(c_{0}^{*}\right)^{\gamma_{0} / \gamma_{i}}+\sum_{i=0}^{k} \alpha_{i}^{(k)} h_{i}
$$


for $k=1, \ldots, T$, where

$$
D_{k}^{(i)}=\delta_{k}^{(i)} e^{-\frac{\rho i}{\gamma_{i}}}\left(\widetilde{M}_{i}\right)^{-1 / \gamma_{i}}
$$

The processes $\left(\delta_{k}^{(i)}\right)_{k=1, \ldots, T ; i=1, \ldots, k}$ and $\left(\alpha_{k}^{(i)}\right)_{k=1, \ldots, T ; i=1, \ldots, k}$ are determined through the following recursive scheme: $\delta_{0}^{(0)}=1, \delta_{1}^{(0)}=\beta_{0}^{(1)}, \delta_{1}^{(1)}=1, \alpha_{1}^{(1)}=1$, and

$$
\begin{aligned}
\alpha_{i}^{(k)} & =\sum_{j=i}^{k-1} \beta_{j}^{(k)} \alpha_{i}^{(j)} ; \quad \alpha_{k}^{(k)}=1, \\
\delta_{k}^{(i)} & =\sum_{j=i}^{k-1} \beta_{j}^{(k)} \delta_{j}^{(i)} ; \quad \delta_{k}^{(k)}=1,
\end{aligned}
$$

for all $k=2, \ldots, T$ and $i=0, \ldots, k-1$. Next, identity (6) in Lemma 2.2 yields

$$
W_{k}^{*}=\sum_{i=0}^{T} F_{k}^{(i)}\left(c_{0}^{*}\right)^{\gamma_{0} / \gamma_{i}}-\sum_{j=k}^{T} E\left[\frac{M_{j}}{M_{k}} \epsilon_{j} \mid \mathcal{G}_{k}\right],
$$

where

$$
F_{k}^{(i)}=\sum_{j=\max (i, k)}^{T} E\left[\frac{M_{j}}{M_{k}} D_{j}^{(i)} \mid \mathcal{G}_{k}\right],
$$

for all $k=1, \ldots, T$ and $i=0, \ldots, T$. Equations 22 and 26 allow to establish a correspondence between the optimal consumption and the wealth as follows. Let $f_{k}: L_{+}^{2}\left(\mathcal{G}_{k}\right) \rightarrow L^{2}\left(\mathcal{G}_{k}\right)$ be defined by $f_{k}(X)=\sum_{i=0}^{T} F_{k}^{(i)} X^{\frac{\gamma_{0}}{\gamma_{i}}}-\sum_{j=k}^{T} E\left[\frac{M_{j}}{M_{k}} \epsilon_{j} \mid \mathcal{G}_{k}\right]$. Since each function of the form $x \mapsto x^{\eta}$ is increasing, for an arbitrary $\eta \geq 0$, it follows that $f_{k}$ is an invertible function. Let us denote by $g_{k}$ the inverse of $f_{k}$, which is defined on $\operatorname{Im}\left(f_{k}\right) \subseteq L^{2}\left(\mathcal{G}_{k}\right)$. Next, consider the function

$$
\psi_{k}(X)=\sum_{i=0}^{k} D_{k}^{(i)} g_{k}^{\frac{\gamma_{0}}{\gamma_{i}}}(X)+\sum_{i=0}^{k} \alpha_{i}^{(k)} h_{i}
$$

and note that $c_{k}^{*}=\psi_{k}\left(W_{k}^{*}\right)$. Observe that $\left(d f_{k}\right)_{X} Y=\sum_{i=0}^{T} F_{k}^{(i)} \frac{\gamma_{0}}{\gamma_{i}} X^{\frac{\gamma_{0}}{\gamma_{i}}-1} Y$, hence, $\left(d f_{k}\right)_{X}=\sum_{i=0}^{T} F_{k}^{(i)} \frac{\gamma_{0}}{\gamma_{i}} X^{\frac{\gamma_{0}}{\gamma_{i}}-1}$ is a multiplication operator. Therefore, we conclude that

$$
\left(d g_{k}\right)_{X} Y=\left(\sum_{i=0}^{T} F_{k}^{(i)} \frac{\gamma_{0}}{\gamma_{i}}\left(g_{k}(X)\right)^{\frac{\gamma_{0}}{\gamma_{i}}-1}\right)^{-1} Y .
$$

The definition of $\psi_{k}$ implies that

$$
\left(d \psi_{k}\right)_{X} Y=\sum_{i=0}^{k} D_{i}^{(k)} \frac{\gamma_{0}}{\gamma_{i}}\left(g_{k}(X)\right)^{\gamma_{0} / \gamma_{i}-1}\left(d g_{k}\right)_{X} Y,
$$


which implies in particular that $\left(d \psi_{k}\right)_{X}$ is a multiplication operator. It is left to check that $0<\left(d \psi_{k}\right)_{W_{k}^{*}} \leq 1$. To this end, note that

$$
\left(d \psi_{k}\right)_{W_{k}^{*}}=\frac{\sum_{i=0}^{k} D_{k}^{(i)} \frac{\gamma_{0}}{\gamma_{i}}\left(c_{0}^{*}\right)^{\frac{\gamma_{0}}{\gamma_{i}}-1}}{\sum_{i=0}^{T} F_{k}^{(i)} \frac{\gamma_{0}}{\gamma_{i}}\left(c_{0}^{*}\right)^{\frac{\gamma_{0}}{\gamma_{i}}-1}}
$$

and the claim follows since $D_{k}^{(i)} \leq F_{k}^{(i)}$, for all $i=0, \ldots, T$ and $k=0, \ldots, T$. Finally, observe that Eqs. 28 and 21 imply that

$$
\psi_{0}\left(\epsilon_{0}\right)+\sum_{i=0}^{T} \sum_{k=i}^{T} E\left[M_{k} D_{k}^{(i)}\right] \psi_{0}^{\gamma_{0} / \gamma_{i}}\left(\epsilon_{0}\right)-\epsilon_{0}-\sum_{k=1}^{T} \sum_{i=1}^{k} E\left[M_{k} \alpha_{i}^{(k)} h_{i}\right]=0,
$$

and thus by differentiating this equation with respect to $\epsilon_{0}$, we obtain that

$$
0<\psi_{0}^{\prime}\left(\epsilon_{0}\right)=\frac{1}{1+\sum_{i=1}^{T} \sum_{k=i}^{T} E\left[M_{k} D_{k}^{(i)}\right] \psi_{0}^{\gamma_{0} / \gamma_{i}-1}\left(\epsilon_{0}\right)}<1 .
$$

(ii) The assertion follows easily by part (i).

\section{General incomplete markets: explicit solution and monotonicity}

In this section we study in more detail an individual's habit-forming utility maximization problem in a general setting of incomplete markets. This general framework requires a more delicate investigation than the one exhibited in the previous section, due to the presence of random endowments, additive habits and general preferences. We will provide an analytic solution to the utility maximization problem through an explicit recursive construction based on the implicit function theorem. We will use this construction to investigate monotonicity properties of the optimal consumption/investment policy.

\subsection{Dynamic programming fails in showing monotonicity}

A conventional approach to tackle the monotonicity problem would be by using dynamic programming (see [27]). Despite that this general methodology provides a useful characterization of the controls involved in the optimization problem, it cannot be directly applied to proving monotonicity in the current setting. We will illustrate this below for the problem of detecting the dependence of the initial consumption $c_{0}\left(\epsilon_{0}\right)$ on the initial endowment $\epsilon_{0}$.

Consider the value function associated with the utility maximization problem (4) viewed as a function of $\epsilon_{0}$ :

$$
V_{0}\left(\epsilon_{0}\right):=\sup _{\pi \in \Pi_{0}} \sum_{k=0}^{T} E\left[u_{k}(c(k, \beta, h, \pi))\right] .
$$

Here, $c(k, \beta, h, \pi):=c_{k}^{\pi}-\sum_{j=0}^{k-1} \beta_{j}^{(k)} c_{j}^{\pi}-h_{k} ; c_{k}^{\pi}$ is defined analogously to (2); and $\Pi_{0}$ denotes the set of all trading strategies $\pi=\left(\pi_{0}, \ldots, \pi_{T-1}, 0\right)$ such that $c(k, \beta, h, \pi) \geq 0$, for all $k=0, \ldots, T$. Note that $c_{0}^{\pi}$ depends on $\epsilon_{0}$, and thus each $c(k, \beta, h, \pi)$ depends on $\epsilon_{0}$, unless $\beta_{0}^{(k)}=0$. Similarly, for each $k=1, \ldots, T$, and fixed portfolio (up to the period 
$k-1) \widehat{\pi}_{o}, \ldots, \widehat{\pi}_{k-1}$, we consider the $\mathcal{G}_{k}-$ measurable random variable:

$$
\begin{aligned}
& V_{k}\left(\epsilon_{0}, \widehat{\pi}_{o}, \ldots, \widehat{\pi}_{k-1}\right) \\
& =\sup _{\pi \in \Pi\left(\widehat{\pi}_{o}, \ldots, \widehat{\pi}_{k-1}\right)} u_{k}(c(k, \beta, h, \pi))+\sum_{j=k+1}^{T} E\left[u_{j}(c(j, \beta, h, \pi)) \mid \mathcal{G}_{k}\right],
\end{aligned}
$$

where $\Pi\left(\widehat{\pi}_{o}, \ldots, \widehat{\pi}_{k-1}\right) \subseteq \Pi_{0}$ denotes the set of all trading strategies of the form $\left(\widehat{\pi}_{o}, \ldots, \widehat{\pi}_{k-1}, \pi_{k}, \ldots, \pi_{T-1}, 0\right)$. Since at the maturity date we have $\pi_{T}=0$, it follows that

$$
V_{T}\left(\epsilon_{0}, \widehat{\pi}_{0}, \ldots, \widehat{\pi}_{T-1}\right)=u(c(T, \beta, h, \widehat{\pi})),
$$

where $\widehat{\pi}=\left(\widehat{\pi}_{0}, \ldots, \widehat{\pi}_{T-1}, 0\right)$. Let us assume for simplicity that the market excludes redundant assets. This implies the existence of a unique optimal portfolio $\pi\left(\epsilon_{0}\right)=$ $\left(\pi_{0}\left(\epsilon_{0}\right), \ldots, \pi_{T}\left(\epsilon_{0}\right)\right)$. Furthermore, it follows (as in Sect. 4.2) that each $\pi_{k}\left(\epsilon_{0}\right)$ is component-wise differentiable with respect to $\epsilon_{0}$. We denote by $\pi_{k}^{\prime}\left(\epsilon_{0}\right)$ the corresponding gradient. Now, recall that $c(0, \beta, h, \pi)=\epsilon_{0}-\pi_{0} \cdot S_{0}$, hence, the dynamic programming principle implies that

$$
\begin{aligned}
V_{0}\left(\epsilon_{0}\right) & =\sup _{\pi_{0}, \Pi\left(\pi_{0}\right) \neq \emptyset}\left\{u\left(\epsilon_{0}-\pi_{0} \cdot S_{0}\right)+E\left[V_{1}\left(\epsilon_{0}, \pi_{0}\right)\right]\right\} \\
& =u\left(\epsilon_{0}-\pi_{0}\left(\epsilon_{0}\right) \cdot S_{0}\right)+E\left[V_{1}\left(\epsilon_{0}, \pi_{0}\left(\epsilon_{0}\right)\right)\right] .
\end{aligned}
$$

Similar arguments yield

$$
\begin{aligned}
V_{k}\left(\epsilon_{0}, \widehat{\pi}_{0}, \ldots, \widehat{\pi}_{k-1}\right)= & \sup _{\pi_{k}, \Pi\left(\widehat{\pi}_{0}, \ldots, \widehat{\pi}_{k-1}, \pi_{k}\right) \neq \emptyset}\left(u_{k}(c(k, \beta, h, \pi))\right. \\
& \left.-E\left[V_{k}\left(\epsilon_{0}, \widehat{\pi}_{0}, \ldots, \widehat{\pi}_{k-1}, \pi_{k}\left(\epsilon_{0}\right)\right) \mid \mathcal{G}_{k}\right]\right),
\end{aligned}
$$

and

$$
\begin{aligned}
V_{k}\left(\epsilon_{0}, \pi_{0}\left(\epsilon_{0}\right), \ldots, \pi_{k-1}\left(\epsilon_{0}\right)\right)= & u_{k}\left(c\left(k, \beta, h, \pi\left(\epsilon_{0}\right)\right)\right) \\
& +E\left[V_{k}\left(\epsilon_{0}, \pi_{0}\left(\epsilon_{0}\right), \ldots, \pi_{k-1}\left(\epsilon_{0}\right), \pi_{k}\left(\epsilon_{0}\right)\right) \mid \mathcal{G}_{k}\right],
\end{aligned}
$$

for all $k=1, \ldots, T$. Observe that $V_{T}\left(\epsilon_{0}, \widehat{\pi}_{0}, \ldots, \widehat{\pi}_{T-1}\right)$ is differentiable with respect to each coordinate, by definition. Therefore, the above recursive relation combined with the differentiability of $\pi\left(\epsilon_{0}\right)$ implies that each $V_{k}\left(\epsilon_{0}, \pi_{0}, \ldots, \pi_{k-1}\right)$ is differentiable with respect to all coordinates. We claim that

$$
V_{0}^{\prime}\left(\epsilon_{0}\right)=u_{0}^{\prime}\left(c_{0}\left(\epsilon_{0}\right)\right)-\sum_{k=1}^{T} \beta_{0}^{(k)} E\left[u_{k}^{\prime}\left(c\left(k, \beta, h, \pi\left(\epsilon_{0}\right)\right)\right)\right],
$$

where $\left(c_{k}\left(\epsilon_{0}\right)\right)_{k=0, \ldots, T}$ denotes the optimal consumption stream. To this end, differentiate Eq. 31 with respect to $\epsilon_{0}$, and observe that

$$
\begin{aligned}
V_{0}^{\prime}\left(\epsilon_{0}\right)= & u^{\prime}\left(c_{0}\left(\epsilon_{0}\right)\right)\left(1-\pi_{0}^{\prime}\left(\epsilon_{0}\right) \cdot S_{0}\right) \\
& +E\left[\frac{\partial V_{1}}{\partial \epsilon_{0}}\left(\epsilon_{0}, \pi_{0}\left(\epsilon_{0}\right)\right)\right]+E\left[\frac{\partial V_{1}}{\partial \pi_{0}}\left(\epsilon_{0}, \pi_{0}\left(\epsilon_{0}\right)\right) \cdot \pi_{0}^{\prime}\left(\epsilon_{0}\right)\right],
\end{aligned}
$$

where

$$
\frac{\partial V_{1}}{\partial \pi_{0}}\left(\epsilon_{0}, \pi_{0}\left(\epsilon_{0}\right)\right)=\left(\frac{\partial V_{1}}{\partial \phi_{0}}\left(\epsilon_{0}, \pi_{0}\left(\epsilon_{0}\right)\right), \frac{\partial V_{1}}{\partial \pi_{0}^{1}}\left(\epsilon_{0}, \pi_{0}\left(\epsilon_{0}\right)\right), \ldots, \frac{\partial V_{1}}{\partial \pi_{0}^{N}}\left(\epsilon_{0}, \pi_{0}\left(\epsilon_{0}\right)\right)\right) .
$$


Since $\pi_{0}\left(\epsilon_{0}\right)$ is the optimal control, the first-order conditions imply that the following equation is satisfied (component-wise):

$$
u_{0}^{\prime}\left(\epsilon_{0}-\pi_{0}\left(\epsilon_{0}\right) \cdot S_{0}\right) S_{0}=E\left[\frac{\partial V_{1}}{\partial \epsilon_{0}}\left(\epsilon_{0}, \pi_{0}\left(\epsilon_{0}\right)\right)\right] .
$$

Therefore, we get

$$
V_{0}^{\prime}\left(\epsilon_{0}\right)=u^{\prime}\left(c_{0}\left(\epsilon_{0}\right)\right)+E\left[\frac{\partial V_{1}}{\partial \epsilon_{0}}\left(\epsilon_{0}, \pi_{0}\left(\epsilon_{0}\right)\right)\right] .
$$

We turn now to computing the random variable $\frac{\partial V_{1}}{\partial \epsilon_{0}}\left(\epsilon_{0}, \pi_{0}\left(\epsilon_{0}\right)\right)$ explicitly. As before, one checks that

$$
\begin{aligned}
& \frac{\partial V_{k}}{\partial \epsilon_{0}}\left(\epsilon_{0}, \pi_{0}\left(\epsilon_{0}\right), \ldots, \pi_{k-1}\left(\epsilon_{0}\right)\right)=-\beta_{0}^{(k)} u^{\prime}\left(c\left(k, \beta, h, \pi\left(\epsilon_{0}\right)\right)\right) \\
& \left.+E\left[\frac{\partial V_{k+1}}{\partial \epsilon_{0}}\left(\epsilon_{0}, \pi_{0}\left(\epsilon_{0}\right), \ldots, \pi_{k-1}\left(\epsilon_{0}\right), \pi_{k}\left(\epsilon_{0}\right)\right)\right) \mid \mathcal{G}_{k}\right],
\end{aligned}
$$

for all $k=0, \ldots, T-1$. By definition, we have

$$
\frac{\partial V_{T}}{\partial \epsilon_{0}}\left(\epsilon_{0}, \pi_{0}\left(\epsilon_{0}\right), \ldots, \pi_{T-1}\left(\epsilon_{0}\right)=-\beta_{0}^{(T)} u_{T}^{\prime}\left(c\left(T, \beta, h, \pi\left(\epsilon_{0}\right)\right)\right) .\right.
$$

Backward induction implies that

$$
E\left[\frac{\partial V_{1}}{\partial \epsilon_{0}}\left(\epsilon_{0}, \pi_{0}\left(\epsilon_{0}\right)\right)\right]=-\sum_{k=1}^{T} \beta_{0}^{(k)} E\left[u_{k}^{\prime}\left(c\left(k, \beta, h, \pi\left(\epsilon_{0}\right)\right)\right)\right] .
$$

This proves the validity of (30). Assume that there are no habits, i.e., $\beta_{j}^{(k)}=0$, and $h_{k}=0$, for all $k=0, \ldots, T$ and $j=0, \ldots, k-1$. Then, (30) takes the form

$$
V_{0}^{\prime}\left(\epsilon_{0}\right)=u_{0}^{\prime}\left(c_{0}\left(\epsilon_{0}\right)\right) \text {. }
$$

Note that the value function $V_{0}\left(\epsilon_{0}\right)$ is concave, since $u_{k}$ is concave, for all $k=0, \ldots, T$, and thus we obtain that $c_{0}^{\prime}\left(\epsilon_{0}\right)=\frac{V_{0}^{\prime \prime}\left(\epsilon_{0}\right)}{u_{0}^{\prime \prime}\left(c_{0}\left(\epsilon_{0}\right)\right)} \geq 0$. However, in the presence of habits, it does not seem feasible to use the (much more complex) dynamic programming principle (30) to derive the required monotonicity of $c_{0}\left(\epsilon_{0}\right)$. Namely, the presence of the cumbersome term

$$
-\sum_{k=1}^{T} \beta_{0}^{(k)} E\left[u_{k}^{\prime}\left(c\left(k, \beta, h, \pi\left(\epsilon_{0}\right)\right)\right)\right],
$$

makes the analysis significantly more complicated and other methods have to be developed. Note also that the other desirable inequality $c_{0}^{\prime}\left(\epsilon_{0}\right) \leq 1$ does not follow from (30) even in the case without habits.

\subsection{Explicit solution and monotonicity}

We provide an explicit solution to the utility maximization problem and study the associated monotonicity issues of the optimal consumption/investment policy, in the setting of arbitrary incomplete markets with a deterministic interest rate, and for arbitrary idiosyncratically (incomplete) markets. We begin with a concise description of the later type of markets. For some related works involving the concept of idiosyncratic source of risk, the reader is 
addressed to Duffie et al. [12], Hendersen [19], Malamud [24] and Malamud and Trubowitz [25].

Definition 4.1 A financial market is called idiosyncratically incomplete, if there exist two filtrations $\left(\mathcal{F}_{k}\right)_{k=0, \ldots, T}$ and $\left(\mathcal{G}_{k}\right)_{k=0, \ldots, T}$ such that:

(i) $\mathcal{F}_{0}=\mathcal{G}_{0}=\{\varnothing, \Omega\}$, and $\mathcal{F}_{k} \subseteq \mathcal{G}_{k}$, for all $k=1, \ldots, T$.

(ii) The market is complete with respect to $\mathcal{F}$, and the endowment stream $\left(\epsilon_{k}\right)_{k=0, \ldots, T}$ is adapted with respect to $\mathcal{G}$.

(iii) For each $k=0, \ldots, T-1$, and an arbitrary random variable $X \in L^{2}\left(\mathcal{F}_{k+1}\right)$, we have

$$
E\left[X \mid \mathcal{G}_{k}\right]=E\left[X \mid \mathcal{F}_{k}\right] .
$$

Lemma 4.1 For an arbitrary idiosyncratically incomplete market, we have

(i) For each $k=1, \ldots, T$, the financial wealth space is given by

$$
\mathcal{L}_{k}=L^{2}\left(\sigma\left(\mathcal{G}_{k-1}, \mathcal{F}_{k}\right)\right),
$$

and, in particular, $P_{k}^{\mathcal{L}}[\cdot]=E\left[\cdot \mid \sigma\left(\mathcal{G}_{k-1}, \mathcal{F}_{k}\right)\right]$.

(ii) The aggregate $S P D$ is a positive process, adapted with respect to $\mathcal{F}$. Moreover, the aggregate SPD coincides with the unique positive SPD that represents the same financial market with the filtration $\mathcal{F}$.

Proof of Lemma 4.1 See the proof of Propositions 4.2 and 4.3 in Malamud and Trubowitz [25].

The main result of this section heavily relies on the following important algebraic identities, leading to a severe simplification of the first order Eq. 7.

Lemma 4.2 For a given incomplete market with a deterministic interest rate, or for an idiosyncratically incomplete market, the first order conditions (7) are equivalent to

$$
P_{\mathcal{L}}^{k}\left[u_{k}^{\prime}\left(c\left(k, \beta, h_{k}\right)\right)\right]=\frac{\widetilde{M}_{k}}{\widetilde{M}_{k t-1}} u_{k-1}^{\prime}\left(c\left(k-1, \beta, h_{k-1}\right)\right),
$$

for all $k=1, \ldots, T$, where the aggregate perturbed $\operatorname{SPD}\left(\widetilde{M}_{k}\right)_{k=1, \ldots, T}$ is defined in (9).

Proof of Lemma 4.2 The proof is based on the following simple observations: For markets with a deterministic interest rate, one checks that the quantities $E\left[\frac{M_{k}}{M_{k-1}} \mid \mathcal{G}_{k-1}\right]$ and $E\left[\frac{\widetilde{M}_{k}}{\widetilde{M}_{k-1}} \mid \mathcal{G}_{k-1}\right]$, are positive and deterministic, for all $k=1, \ldots, T$; for idiosyncratically incomplete markets, the aggregate perturbed SPD satisfies: $\widetilde{M}_{k} \in \mathcal{F}_{k}$, for all $k=1, \ldots, T$. We provide a unified proof for both cases. For the sake of brevity, we set $\widehat{c}_{k}:=c\left(k, \beta, h_{k}\right)$, for $k=0, \ldots, T$. Consider Eq. 7 for $k=T$ :

$$
P_{\mathcal{L}}^{T}\left[u_{T}^{\prime}\left(\widehat{c}_{T}\right)\right]=\frac{M_{T}}{M_{T-1}}\left(u_{T-1}^{\prime}\left(\widehat{c}_{T-1}\right)-\beta_{T-1}^{(T)} E\left[u_{T}^{\prime}\left(\widehat{c}_{T}\right) \mid \mathcal{G}_{T-1}\right]\right) .
$$

An application of the conditional expectation $E\left[\cdot \mid \mathcal{G}_{T-1}\right]$ to both sides of this equation yields

$$
E\left[u_{T}^{\prime}\left(\widehat{c}_{T}\right) \mid \mathcal{G}_{T-1}\right]=\frac{E\left[M_{T} \mid \mathcal{G}_{T-1}\right]}{M_{T-1}+\beta_{T-1}^{(T)} E\left[M_{T} \mid \mathcal{G}_{T-1}\right]} u_{T-1}^{\prime}\left(\widehat{c}_{T-1}\right),
$$


and by plugging this back into (33), one concludes that (32) holds for $k=T$. Next, in the idiosyncratically incomplete case, one can combine the preceding formula with property (i) in Lemma 4.1 (in the deterministic interest rate case, one can exploit the fact that $\frac{M_{T-1}}{M_{T-1}+\beta_{T-1}^{(T)} E\left[M_{T} \mid \mathcal{G}_{T-1}\right]}$ is a positive constant), to check that Eq. 7 admits the following form, for $k=T-1$,

$$
\begin{aligned}
& \frac{M_{T-1}}{M_{T-1}+\beta_{T-1}^{(T)} E\left[M_{T} \mid \mathcal{G}_{T-1}\right]} P_{\mathcal{L}}^{T-1}\left[u_{T-1}^{\prime}\left(\widehat{c}_{T-1}\right)\right] \\
& =\frac{M_{T-1}}{M_{T-2}}\left(u_{T-2}^{\prime}\left(\widehat{c}_{T-2}\right)-E\left[\left(\beta_{T-2}^{(T-1)}+\beta_{T-2}^{(T)} \frac{\widetilde{M}_{T}}{\widetilde{M}_{T-1}}\right) u_{T-1}^{\prime}\left(\widehat{c}_{T-1}\right) \mid \mathcal{G}_{T-1}\right]\right) .
\end{aligned}
$$

Now, one can multiply the above equation by

$$
E\left[\beta_{T-2}^{(T-1)}+\beta_{T-2}^{(T)} \frac{\tilde{M}_{T}}{\widetilde{M}_{T-1}} \mid \mathcal{G}_{T-1}\right] \frac{M_{T-1}+\beta_{T-1}^{(T)} E\left[M_{T} \mid \mathcal{G}_{T-1}\right]}{M_{T-1}},
$$

apply then the conditional expectation $E\left[\cdot \mid \mathcal{G}_{T-2}\right]$ to both sides of the resulted equation, and proceed as follows to conclude the validity of (32), for $k=T-1$ : In the idiosyncratically incomplete case, one can use property (iii) in Definition 4.1 and the identity $X P_{L}^{k}[Y]=$ $P_{L}^{k}[X Y]$, which holds for all $Y \in L^{2}\left(\mathcal{G}_{T}\right), X \in L^{2}\left(\mathcal{F}_{k}\right)$, and all $k=1, \ldots, T$; in the deterministic interest rate case, one can use the fact that $E\left[\beta_{T-2}^{(T-1)}+\beta_{T-2}^{(T)} \frac{\widetilde{M}_{T}}{\bar{M}_{T-1}} \mid \mathcal{G}_{T-1}\right]$ and $\frac{M_{T-1}+\beta_{T-1}^{(T)} E\left[M_{T} \mid \mathcal{G}_{T-1}\right]}{M_{T-1}}$ are positive constants. The rest of the proof follows by similar arguments and induction.

Now, we present the main result of this section.

Theorem 4.1 Consider the utility maximization problem (4) in the setting of an incomplete market with a deterministic interest rate, or an idiosyncratically incomplete market. Then, we have

$$
\begin{aligned}
c_{T}^{*} & =\psi_{T}\left(W_{T}^{*}\right), \\
c_{k}^{*} & =\psi_{k}\left(W_{k}^{*}, c_{k-1}^{*}, \ldots, c_{0}^{*}\right),
\end{aligned}
$$

for $k=1, \ldots, T-1$, and

$$
\begin{aligned}
c_{0}^{*} & =\psi_{0}\left(\epsilon_{0}\right), \\
W_{k}^{*} & =\eta_{k}\left(c_{k-1}^{*}, \ldots, c_{0}^{*}\right),
\end{aligned}
$$

for $k=2, \ldots, T$, and

$$
W_{1}^{*}=\eta_{1}\left(c_{0}^{*}\right),
$$

where the functions $\psi_{T},\left(\psi_{k}\right)_{k=0, \ldots, T-1}$ and $\left(\eta_{k}\right)_{k=1, \ldots, T}$ are given in (34), (53), (55) and (57). Furthermore, a monotonicity property of the optimal consumption holds in the following form: The differential of $\psi_{k}$, for $k=1, \ldots, T$, with respect to $W_{k}$ is a multiplication operator that satisfies:

$$
0<\frac{\partial \psi_{k}}{\partial W_{k}} \leq \frac{1}{1+\sum_{j=k+1}^{T}\left(\sum_{i=k}^{j} \beta_{k}^{i} \beta_{i}^{i+1} \cdots \beta_{j-1}^{j}\right) E\left[\frac{M_{j}}{M_{k}} \mid \mathcal{G}_{k}\right]},
$$


for $k=1, \ldots, T$, and

$$
0<\psi_{0}^{\prime}\left(\epsilon_{0}\right) \leq \frac{1}{1+\sum_{j=1}^{T}\left(\sum_{i=0}^{j} \beta_{0}^{i} \beta_{i}^{i+1} \cdots \beta_{j-1}^{j}\right) E\left[M_{j} \mid \mathcal{G}_{k}\right]}
$$

Remark As in the proof of Lemma 4.2, we present a unified approach that treats both markets with a deterministic interest rate and idiosyncratically incomplete markets. As one can notice, the additive habits structure in our model generate an essential distinction between the interaction of the optimal consumption/investment rules in the last two periods. The reason for this comes from the fact that the consumption at the maturity has no impact on future consumption. Therefore, we focus on proving the statement for the periods $k=T-2, T-1, T$, which constitutes the core of Theorem 4.1. The rest is then concisely explained, and can be proved by induction, without any further machinery.

Proof of Theorem 4.1 To simplify notations, we set $\widehat{c}_{k}^{*}:=c_{k}^{*}-\sum_{l=0}^{k-1} \beta_{l}^{(k)} c_{l}^{*}-h_{k}$, for all $k=0, \ldots, T$. For an arbitrary consumption stream $\left(c_{k}\right)_{k=0, \ldots, T}$, as in (3), we denote $\widehat{c}_{k}:=c(k, \beta, h)=c_{k}-\sum_{l=0}^{k-1} \beta_{l}^{(k)} c_{l}-h_{k}$, for all $k=0, \ldots, T$.

The case $k=T$. Let $\psi_{T}: L^{2}\left(\mathcal{G}_{T}\right) \rightarrow L^{2}\left(\mathcal{G}_{T}\right)$ be a function defined by

$$
\psi_{T}(X):=\epsilon_{T}+X .
$$

Observe that relation (8), for $k=T$, implies that $c_{T}^{*}=\psi_{T}\left(W_{T}^{*}\right)$. Furthermore, $d \psi_{T}=1$. Next, consider the function

$$
\begin{aligned}
f_{T}\left(c_{0}, \ldots, c_{T-1}, W_{T}\right)= & P_{\mathcal{L}}^{T}\left[u_{T}^{\prime}\left(\psi_{T}\left(W_{T}\right)-\sum_{l=0}^{T-1} \beta_{l}^{(T)} c_{l}-h_{T}\right)\right] \\
& -\frac{\widetilde{M}_{T}}{\widetilde{M}_{T-1}} u_{T-1}^{\prime}\left(\widehat{c}_{T-1}\right),
\end{aligned}
$$

defined on the set $B_{T}^{\prime} \subseteq L^{2}\left(\mathcal{G}_{0}\right) \times \cdots \times L^{2}\left(\mathcal{G}_{T-1}\right) \times \mathcal{L}_{T}$ consisting of all tuples $\left(c_{0}, \ldots, c_{T-1}, W_{T}\right)$ such that $\psi_{T}\left(W_{T}\right)-\sum_{l=0}^{T-1} \beta_{l}^{(T)} c_{l}-h_{T} \geq 0$ and $\widehat{c}_{T-1} \geq 0, P-$ a.s. Note that Lemma 4.2 implies that $f_{T}\left(c_{0}^{*}, \ldots, c_{T-1}^{*}, W_{T}^{*}\right)=0$. Furthermore, the differential of $f_{T}$ with respect to $W_{T}$, at some $\left(c_{0}, \ldots, c_{T-1}, W_{T}\right) \in \operatorname{int}\left(B_{T}\right), \frac{\partial f_{T}}{\partial W_{T}}:=\frac{\partial f_{T}}{\partial W_{T}}\left(c_{0}, \ldots, c_{T-1}, W_{T}\right)$, applied on some vector $V \in \mathcal{L}_{T}, V \neq 0$, is given by

$$
\frac{\partial f_{T}}{\partial W_{T}} V=P_{\mathcal{L}}^{T}\left[u_{T}^{\prime \prime}\left(\psi_{T}\left(W_{T}\right)-\sum_{l=0}^{T-1} \beta_{l}^{(T)} c_{l}-h_{T}\right) V\right] .
$$

Note that part (iii) of Lemma 2.1 yields

$$
\begin{aligned}
& E\left[V \frac{\partial f_{T}}{\partial W_{k}}\left(c_{0}, \ldots, c_{T-1}, W_{T}\right)\right. \\
& \left.=E \mid \mathcal{G}_{T-1}\right] \\
& =E\left[u_{T}^{\prime \prime}\left(\psi_{T}\left(W_{T}\right)-\sum_{l=0}^{T-1} \beta_{l}^{(T)} c_{l}-h_{T}\right) V^{2} \mid \mathcal{G}_{T-1}\right]<0,
\end{aligned}
$$

where the last inequality follows by the concavity of $u_{T}$. This in particular implies that the operator

$$
\frac{\partial f_{T}}{\partial W_{T}\left(c_{0}^{*}, \ldots, c_{T-1}^{*}, W_{T}^{*}\right)}: \mathcal{L}_{T} \rightarrow \mathcal{L}_{T}
$$


which is given by

$$
\frac{\partial f_{T}}{\partial W_{T}\left(c_{0}^{*}, \ldots, c_{T-1}^{*}, W_{T}^{*}\right)} \underset{ }{ } V:=P_{T}^{\mathcal{L}}\left[u_{T}^{\prime \prime}\left(\widehat{c}_{T}^{*}\right) V\right]
$$

is non-degenerate. The implicit function theorem implies that there exists an open neighborhood $D_{T-1}$ of $\left(c_{0}^{*}, \ldots, c_{T-1}^{*}\right)$ (in the $L^{2}\left(\mathcal{G}_{0}\right) \times \cdots \times L^{2}\left(\mathcal{G}_{T-1}\right)$-topology) and a unique $C^{1}$-function $\eta_{T}: D_{T-1} \rightarrow \mathcal{L}_{T}$ such that

$$
f_{T}\left(c_{0}, \ldots, c_{T-1}, \eta_{T}\left(c_{0}, \ldots, c_{T-1}\right)\right)=0,
$$

for all $\left(c_{0}, \ldots, c_{T-1}\right) \in D_{T-1}$. We will compute now certain differentials that will be crucial for proving the statement in the phase: $k=T-1$. A differentiation of (36) with respect to $c_{T-1}$ and $c_{k}, k=0, \ldots, T-2$, combined with item (ii) of Lemma 2.1, implies the validity of the following identities,

$$
\begin{aligned}
& P_{\mathcal{L}}^{T}\left[u_{T}^{\prime \prime}\left(\psi_{T}\left(\eta_{T}\left(c_{0}, \ldots, c_{T-1}\right)\right)-\sum_{l=0}^{T-1} \beta_{l}^{(T)} c_{l}-h_{T}\right)\left(\frac{\partial \eta_{T}}{\partial c_{T-1}} V-\beta_{T-1}^{(T)} V\right)\right] \\
& =\frac{\widetilde{M}_{T}}{\widetilde{M}_{T-1}} u_{T-1}^{\prime \prime}\left(\widehat{c}_{T-1}\right) V
\end{aligned}
$$

for all random variables $V \in L^{2}\left(G_{T-1}\right)$, and

$$
\begin{aligned}
& P_{\mathcal{L}}^{T}\left[u_{T}^{\prime \prime}\left(\psi_{T}\left(\eta_{T}\left(c_{0}, \ldots, c_{T-1}\right)\right)-\sum_{l=0}^{T-1} \beta_{l}^{(T)} c_{l}-h_{T}\right)\left(\frac{1}{W} \frac{\partial \eta_{T}}{\partial c_{k}} W-\beta_{k}^{(T)}\right)\right] \\
& \quad=-\beta_{k}^{(T-1)} \frac{\widetilde{M}_{T}}{\widetilde{M}_{T-1}} u_{T-1}^{\prime \prime}\left(\widehat{c}_{T-1}\right),
\end{aligned}
$$

for all non-vanishing random variables $W \in L^{2}\left(G_{k}\right), k=0, \ldots, T-2$, where $\frac{\partial \eta_{T}}{\partial c_{T-1}}:=$ ${\frac{\partial \eta_{T}}{\partial c_{T-1}}\left(c_{0}, \ldots, c_{T-1}\right)}$ and $\frac{\partial \eta_{T}}{\partial c_{k}}:=\frac{\partial \eta_{T}}{\partial c_{k}}\left(c_{0}, \ldots, c_{T-1}\right)$. Therefore, by multiplying Eqs. 37 and 38 by $\beta_{k}^{(T-1)}$ and $V$ respectively, applying property (ii) in Lemma 2.1, and summing up these equations, we obtain

$$
\begin{aligned}
& P_{\mathcal{L}}^{T}\left[u_{T}^{\prime \prime}\left(\psi_{T}\left(\eta_{T}\left(c_{0}, \ldots, c_{T-1}\right)\right)-\sum_{l=0}^{T-1} \beta_{l}^{(T)} c_{l}-h_{T}\right) \cdot\left(\beta_{k}^{(T-1)} \frac{\partial \eta_{T}}{\partial c_{T-1}} V+\frac{V}{W} \frac{\partial \eta_{T}}{\partial c_{k}} W\right)\right] \\
& =P_{\mathcal{L}}^{T}\left[u_{T}^{\prime \prime}\left(\psi_{T}\left(\eta_{T}\left(c_{0}, \ldots, c_{T-1}\right)\right)-\sum_{l=0}^{T-1} \beta_{l}^{(T)} c_{l}-h_{T}\right)\left(\beta_{k}^{(T-1)} \beta_{T-1}^{(T)}+\beta_{k}^{(T)}\right) V\right],
\end{aligned}
$$

and thus by part (iv) of Lemma 2.1, it follows that

$$
\frac{\partial \eta_{T}}{\partial c_{T-1}} V=\frac{1}{\beta_{k}^{(T-1)}}\left(\beta_{k}^{(T-1)} \beta_{T-1}^{(T)}+\beta_{k}^{(T)}-\frac{1}{W} \frac{\partial \eta_{T}}{\partial c_{k}} W\right) V .
$$

By employing similar arguments, one can check that Eq. 38 implies that

$$
\frac{\partial \eta_{T}}{\partial c_{k}} W=\left(\beta_{k}^{(T)}+\frac{\beta_{k}^{(T-1)}}{\beta_{m}^{(T-1)}}\left(\frac{1}{Y} \frac{\partial \eta_{T}}{\partial c_{m}} Y-\beta_{m}^{(T)}\right)\right) W,
$$


for all $k, m=0, \ldots, T-2, W \in L^{2}\left(\mathcal{G}_{k-1}\right)$ and all non-vanishing random variables $Y \in$ $L^{2}\left(\mathcal{G}_{m-1}\right)$. In particular we deduce that $\frac{\partial \eta_{T}}{\partial c_{T-1}}$, and $\frac{\partial \eta_{T}}{\partial c_{k}}$, for $k=0, \ldots, T-2$, are multiplication operators (and thus can be treated as random variables). That is, we can rewrite (39) as

$$
\frac{\partial \eta_{T}}{\partial c_{T-1}}=\frac{1}{\beta_{k}^{(T-1)}}\left(\beta_{k}^{(T-1)} \beta_{T-1}^{(T)}+\beta_{k}^{(T)}-\frac{\partial \eta_{T}}{\partial c_{k}}\right) .
$$

The case $k=T-1$. We set the function

$$
\begin{aligned}
g_{T-1}: D_{T-1} \times \mathcal{L}_{T-1} \rightarrow L^{2} & \left(\mathcal{G}_{T-1}\right) \\
g_{T-1}\left(c_{0}, \ldots, c_{T-1}, W_{T-1}\right)= & c_{T-1}+E\left[\frac{M_{T}}{M_{T-1}} \eta_{T}\left(c_{0}, \ldots, c_{T-1}\right) \mid \mathcal{G}_{T-1}\right] \\
& -W_{T-1}-\epsilon_{T-1},
\end{aligned}
$$

and note that by $(8), g_{T-1}\left(c_{0}^{*}, \ldots, c_{T-1}^{*}, W_{T-1}^{*}\right)=0$. Let

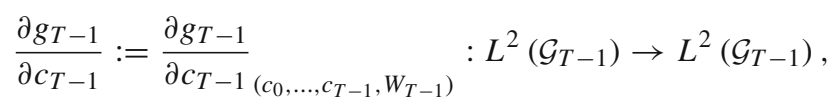

be the derivative, which is given by

$$
\frac{\partial g_{T-1}}{\partial c_{T-1}} V=V+E\left[\frac{M_{T}}{M_{T-1}} \frac{\partial \eta_{T}}{\partial c_{T-1}} V \mid \mathcal{G}_{T-1}\right],
$$

for all $V \in L^{2}\left(\mathcal{G}_{T-1}\right)$. Observe that

$$
E\left[V \frac{\partial g_{T-1}}{\partial c_{T-1}} V \mid \mathcal{G}_{T-1}\right]=V^{2}+E\left[\frac{M_{T}}{M_{T-1}} V \frac{\partial \eta_{T}}{\partial c_{T-1}} V \mid \mathcal{G}_{T-1}\right] .
$$

Let us show that $E\left[\frac{M_{T}}{M_{T-1}} V \frac{\partial \eta_{T}}{\partial c_{T-1}} V \mid \mathcal{G}_{T-1}\right]>0$, for $V \neq 0$. By (41), we have

$$
\begin{aligned}
& E\left[\frac{M_{T}}{M_{T-1}} V \frac{\partial \eta_{T}}{\partial c_{T-1}} V \mid \mathcal{G}_{T-1}\right] \\
& \quad=\beta_{T-1}^{(T)} E\left[\frac{M_{T}}{M_{T-1}} \mid \mathcal{G}_{T-1}\right] V^{2}+\frac{1}{\beta_{k}^{(T-1)}} E\left[\frac{M_{T}}{M_{T-1}}\left(\beta_{k}^{(T)}-\frac{\partial \eta_{T}}{\partial c_{k}}\right) \mid \mathcal{G}_{T-1}\right] V^{2}
\end{aligned}
$$

Recall that $E\left[\frac{M_{T}}{M_{T-1}} \mid \mathcal{G}_{T-1}\right]$ denotes the interest rate at period $T-1$, and thus it is in particular a positive quantity. Thereby, it suffices to show that

$$
E\left[\frac{M_{T}}{M_{T-1}}\left(\beta_{k}^{(T)}-\frac{\partial \eta_{T}}{\partial c_{k}}\right) \mid \mathcal{G}_{T-1}\right]>0 .
$$

In virtue of (38), this is equivalent to showing that

$$
\begin{aligned}
& \frac{1}{\beta_{k}^{(T-1)} u_{T-1}^{\prime \prime}\left(\widehat{c}_{T-1}\right)} E\left[\frac{M_{T}}{\widetilde{M}_{T}} \frac{M_{T-1}}{\widetilde{M}_{T-1}}\left(\beta_{k}^{(T)}-\frac{\partial \eta_{T}}{\partial c_{k}}\right)\right. \\
& \quad \times P_{\mathcal{L}}^{T}\left[u_{T}^{\prime \prime}\left(\psi_{T}\left(\eta_{T}\left(c_{0}, \ldots, c_{T-1}\right)\right)-\sum_{l=0}^{T-1} \beta_{l}^{(T)} c_{l}-h_{T}\right)\right. \\
& \left.\left.\quad \times\left(\beta_{k}^{(T)}-\frac{\partial \eta_{T}}{\partial c_{k}}\right)\right] \mid \mathcal{G}_{T-1}\right]>0 .
\end{aligned}
$$


To this end, let us first prove it for the deterministic interest rate case, and then for idiosyncratically incomplete markets. Note that in the former case, the quantities $\frac{M_{k}}{\widetilde{M}_{K}}, k=1, \ldots, T$ are positive numbers, and hence, since $\beta_{k}^{(T)}-\frac{1}{W} \frac{\partial \eta_{T}}{\partial c_{k}} W \in \mathcal{L}_{T}$, part (iii) of Lemma 2.1, implies that (42) is equivalent to

$$
E\left[u_{T}^{\prime \prime}\left(\psi_{T}\left(\eta_{T}\left(c_{0}, \ldots, c_{T-1}\right)\right)-\sum_{l=0}^{T-1} \beta_{l}^{(T)} c_{l}-h_{T}\right)\left(\beta_{k}^{(T)}-\frac{\partial \eta_{T}}{\partial c_{k}}\right)^{2} \mid \mathcal{G}_{T-1}\right]<0,
$$

which holds true since $u_{T}$ is a concave function. For idiosyncratically incomplete markets, notice first that the ratio $\frac{M_{k}}{\widetilde{M}_{k}}$ is positive, for all $k=1, \ldots, T$. Next, since $P_{k}^{\mathcal{L}}[\cdot]=$ $E\left[\cdot \mid \sigma\left(\mathcal{G}_{k-1}, \mathcal{F}_{k}\right)\right]$, it follows that $P_{k}^{\mathcal{L}}[X Y]=X P_{k}^{\mathcal{L}}[Y]$ holds, for all $Y \in L^{2}\left(\mathcal{G}_{T}\right)$ and $X \in \mathcal{L}_{k}=L^{2}\left(\sigma\left(\mathcal{G}_{k-1}, \mathcal{F}_{k}\right)\right)$. By exploiting these observations, one easily checks that (42) is equivalent to

$$
\begin{aligned}
& E\left[u_{T}^{\prime \prime}\left(\psi_{T}\left(\eta_{T}\left(c_{0}, \ldots, c_{T-1}\right)\right)-\sum_{l=0}^{T-1} \beta_{l}^{(T)} c_{l}-h_{T}\right)\right. \\
& \left.\times\left(\beta_{k}^{(T)}-\frac{\partial \eta_{T}}{\partial c_{k}}\right)^{2} \mid \sigma\left(\mathcal{G}_{k-1}, \mathcal{F}_{k}\right)\right]<0,
\end{aligned}
$$

which is evidently satisfied. Therefore, it follows that the operator

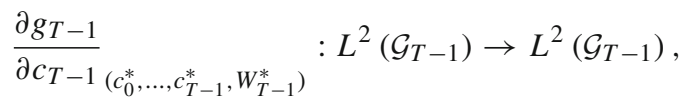

which is given by

$$
\begin{aligned}
& \frac{\partial g_{T-1}}{\partial c_{T-1}}\left(c_{0}^{*}, \ldots, c_{T-1}^{*}, W_{T-1}^{*}\right) \\
& =V+E\left[\frac{M_{T}}{M_{T-1}} \frac{\partial \eta_{T}}{\partial c_{T-1}}\left(c_{0}^{*}, \ldots, c_{T-2}^{*}, W_{T-1}^{*}\right)\right. \\
& \left.=V \mid \mathcal{G}_{T-1}\right]
\end{aligned}
$$

is non-degenerate. The implicit function theorem implies that there exists an open neighborhood $B_{T-1}$ of $\left(c_{0}^{*}, \ldots, c_{T 2}^{*}, W_{T-1}^{*}\right)$ (in the $L^{2}\left(\mathcal{G}_{0}\right) \times \cdots \times L^{2}\left(\mathcal{G}_{T-2}\right) \times \mathcal{L}_{T-1}$-topology) and a unique $C^{1}$-function $\psi_{T-1}: B_{T-1} \rightarrow L^{2}\left(\mathcal{G}_{T-2}\right)$ such that

$$
\begin{aligned}
& g_{T-1}\left(c_{0}, \ldots, c_{T-2}, \psi_{T-1}\left(c_{0}, \ldots, c_{T-2}, W_{T-1}\right), W_{T-1}\right)=\Psi_{T-1}\left(c_{0}, \ldots, c_{T-2}, W_{T-1}\right) \\
& +E\left[\frac{M_{T}}{M_{T-1}} \eta_{T}\left(c_{0}, \ldots, c_{T-2}, \psi_{T-1}\left(c_{0}, \ldots, c_{T-2}, W_{T-1}\right)\right) \mid \mathcal{G}_{T-1}\right]-W_{T-1}-\epsilon_{T-1}=0,
\end{aligned}
$$

for all $\left(c_{0}, \ldots, c_{T 2}, W_{T-1}\right) \in B_{T-1}$. By differentiating the above equation with respect to $W_{T-1}$ and $c_{k}, k=0, \ldots, T-2$, and using the fact that both $\frac{\partial \eta_{T}}{\partial c_{T-1}}$ and $\frac{\partial \eta_{T}}{\partial c_{k}}$ are multiplicative operators, we obtain that both $\frac{\partial \psi_{T-1}}{\partial W_{T-1}}:=\frac{\partial \psi_{T-1}}{\partial W_{T-1}\left(c_{0}, \ldots, c_{T-2}, W_{T-1}\right)}$ and $\frac{\partial \psi_{T-1}}{\partial c_{k}}:=$ $\frac{\partial \psi_{T-1}}{\partial c_{k}}\left(c_{0}, \ldots, c_{T-2}, W_{T-1}\right)$ are multiplicative operators given explicitly by

$$
\left.\frac{\partial \psi_{T-1}}{\partial W_{T-1}}=\frac{1}{1+E\left[\frac{M_{T}}{M_{T-1}} \frac{\partial \eta_{T}}{\partial c_{T-1}}\left(c_{0}, \ldots, c_{T-2}, \psi_{T-1}\left(c_{0}, \ldots, c_{T-2}, W_{T-1}\right), W_{T-1}\right)\right.} \mid \mathcal{G}_{T-1}\right],
$$


and

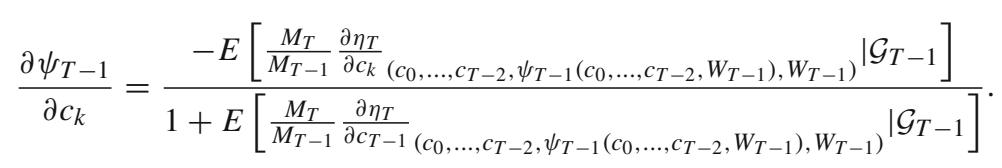

Unless unavoidable, we will henceforth omit the indexes in the derivatives. Next, we define the function,

$$
\begin{aligned}
& f_{T-1}\left(c_{0}, \ldots, c_{T-2}, W_{T-1}\right)=-\frac{\widetilde{M}_{T-1}}{\widetilde{M}_{T-2}} u_{T-2}^{\prime}\left(\widehat{c}_{T-2}\right) \\
& +P_{\mathcal{L}}^{T-1}\left[u_{T-1}^{\prime}\left(\psi_{T-1}\left(c_{0}, \ldots, c_{T-2}, W_{T-1}\right)-\sum_{k=0}^{T-2} \beta_{k}^{(T-1)} c_{k}-h_{T-1}\right)\right],
\end{aligned}
$$

on the set $B_{T-1}$, which consists of all tuples $\left(c_{0}, \ldots, c_{T-2}, W_{T-1}\right) \in L^{2}\left(\mathcal{G}_{0}\right) \times \cdots \times$ $L^{2}\left(\mathcal{G}_{T-2}\right) \times \mathcal{L}_{T-1}$ such that $\psi_{T-1}\left(c_{0}, \ldots, c_{T-2}, W_{T-1}\right)-\sum_{k=0}^{T-2} \beta_{k}^{(T-1)} c_{k}-h_{T-1} \geq 0$ and $\widehat{c}_{T-2} \geq 0$. Observe that

$$
\begin{aligned}
& \frac{\partial f_{T-1}}{\partial W_{T-1}} V \\
& \quad=P_{\mathcal{L}}^{T-1}\left[u_{T-1}^{\prime \prime}\left(\psi_{T-1}\left(c_{0}, \ldots, c_{T-2}, W_{T-1}\right)-\sum_{k=0}^{T-2} \beta_{k}^{(T-1)} c_{k}-h_{T-1}\right) \frac{\partial \psi_{T-1}}{\partial W_{T-1}} V\right],
\end{aligned}
$$

for all $V \in \mathcal{L}_{T-1}$. By item (iii) in Lemma 2.1, it follows that

$$
\begin{aligned}
E\left[V \frac{\partial f_{T-1}}{\partial W_{T-1}} V \mid \mathcal{G}_{T-2}\right]= & E\left[u_{T-1}^{\prime \prime}\left(\psi_{T-1}\left(c_{0}, \ldots, c_{T-2}, W_{T-1}\right)-\sum_{k=0}^{T-2} \beta_{k}^{(T-1)} c_{k}-h_{T-1}\right)\right. \\
& \left.\times \frac{\partial \psi_{T-1}}{\partial W_{T-1}} V^{2} \mid \mathcal{G}_{T-2}\right]<0,
\end{aligned}
$$

where the last inequality follows by the concavity of $u_{T-1}$ and the fact that $\frac{\partial \psi_{T-1}}{\partial W_{T-1}}>0$, which holds due to (44). Therefore, we conclude that the differential

$$
\frac{\partial f_{T-1}}{\partial W_{T-1}\left(c_{0}^{*}, \ldots, c_{T-2}^{*}, W_{T-1}^{*}\right)}: \mathcal{L}_{T-1} \rightarrow \mathcal{L}_{T-1},
$$

which is given by

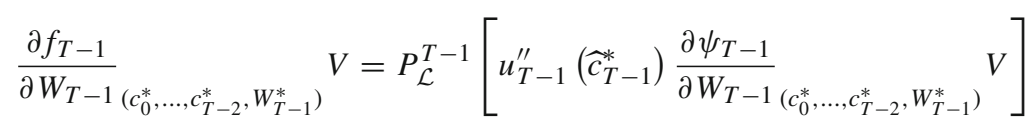

is non-degenerate. The implicit function theorem implies the existence of an open neighborhood $D_{T-2}$ of $\left(c_{0}^{*}, \ldots, c_{T-2}^{*}\right)$ (in the $L^{2}\left(\mathcal{G}_{0}\right) \times \cdots \times L^{2}\left(\mathcal{G}_{T-2}\right)$-topology) and a unique $C^{1}$-function $\eta_{T-1}\left(c_{0}, \ldots, c_{T-2}\right): D_{T-2} \rightarrow \mathcal{L}_{T-1}$, such that

$$
f_{T-1}\left(c_{0}, \ldots, c_{T-2}, \eta_{T-1}\left(c_{0}, \ldots, c_{T-2}\right)\right)=0,
$$

for all $\left(c_{0}, \ldots, c_{T-2}\right) \in D_{T-2}$. We shall compute now some differentials and establish related identities that we will find useful in the subsequence phases. By differentiating the 
above equation with respect to $c_{T-2}$ and then $c_{k}, k=0, \ldots, T-3$, one checks that the following is satisfied:

$$
\begin{aligned}
& P_{\mathcal{L}}^{T-1}\left[u_{T-1}^{\prime \prime}\left(\psi_{T-1}\left(c_{0}, \ldots, c_{T-2}, W_{T-1}\right)-\sum_{k=0}^{T-2} \beta_{k}^{(T-1)} c_{k}-h_{T-1}\right)\right. \\
& \left.\quad \times\left(\frac{\partial \psi_{T-1}}{\partial c_{T-2}} \cdot V+\frac{\partial \psi_{T-1}}{\partial W_{T-1}} \cdot \frac{\partial \eta_{T-1}}{\partial c_{T-2}} V-\beta_{T-2}^{(T-1)} \cdot V\right)\right]=\frac{\widetilde{M}_{T-1}}{\widetilde{M}_{T-2}} u_{T-2}^{\prime}\left(\widehat{c}_{T-2}\right) \cdot V,
\end{aligned}
$$

for all $V \in L^{2}\left(\mathcal{G}_{T-2}\right)$, and

$$
\begin{aligned}
& P_{\mathcal{L}}^{T-1}\left[u_{T-1}^{\prime \prime}\left(\psi_{T-1}\left(c_{0}, \ldots, c_{T-2}, W_{T-1}\right)-\sum_{k=0}^{T-2} \beta_{k}^{(T-1)} c_{k}-h_{T-1}\right)\right. \\
& \left.\times\left(\frac{\partial \psi_{T-1}}{\partial c_{k}}+\frac{\partial \psi_{T-1}}{\partial W_{T-1}} \cdot \frac{1}{W} \frac{\partial \eta_{T-1}}{\partial c_{k}} W-\beta_{k}^{(T-1)}\right)\right]=-\beta_{k}^{(T-2)} \frac{\widetilde{M}_{T-1}}{\widetilde{M}_{T-2}} u_{T-2}^{\prime}\left(\widehat{c}_{T-2}\right),
\end{aligned}
$$

for all non-vanishing vectors $W \in L^{2}\left(\mathcal{G}_{k}\right), k=0, \ldots, T-3$. Similarly as in (39) and (40), by part (iv) of Lemma 2.1, we obtain that

$$
\begin{aligned}
& \beta_{k}^{(T-2)}\left(\frac{\partial \psi_{T-1}}{\partial c_{T-2}} \cdot V+\frac{\partial \psi_{T-1}}{\partial W_{T-1}} \cdot \frac{\partial \eta_{T-1}}{\partial c_{T-2}} V-\beta_{T-2}^{(T-1)} \cdot V\right) \\
& +\quad\left(\frac{\partial \psi_{T-1}}{\partial c_{k}}+\frac{1}{W} \frac{\partial \psi_{T-1}}{\partial W_{T-1}} \cdot \frac{\partial \eta_{T-1}}{\partial c_{k}} W-\beta_{k}^{(T-1)}\right) \cdot V=0
\end{aligned}
$$

or equivalently

$$
\begin{aligned}
\frac{\partial \eta_{T-1}}{\partial c_{T-2}}= & \left(\beta_{T-2}^{(T-1)}-\frac{\partial \psi_{T-1}}{\partial c_{T-2}}-\frac{1}{\beta_{k}^{(T-2)}}\left(\frac{\partial \psi_{T-1}}{\partial c_{k}}\right.\right. \\
& \left.\left.+\frac{1}{W} \frac{\partial \psi_{T-1}}{\partial W_{T-1}} \cdot \frac{\partial \eta_{T-1}}{\partial c_{k}} W-\beta_{k}^{(T-1)}\right)\right) \frac{1}{\frac{\partial \psi_{T-1}}{\partial W_{T-1}}}
\end{aligned}
$$

We turn now to a simplification of some terms in the brackets above,

$$
\begin{aligned}
\beta_{T-2}^{(T-1)}-\frac{\partial \psi_{T-1}}{\partial c_{T-2}}= & \frac{\beta_{T-2}^{(T-1)}+E\left[\frac{M_{T}}{M_{T-1}}\left(\beta_{T-2}^{(T-1)} \frac{\partial \eta_{T}}{\partial c_{T-1}}+\frac{\partial \eta_{T}}{\partial c_{T-2}}\right) \mid \mathcal{G}_{T-1}\right]}{1+E\left[\frac{M_{T}}{M_{T-1}} \frac{\partial \eta_{T}}{\partial c_{T-1}} \mid \mathcal{G}_{T-1}\right]} \\
& =\frac{\beta_{T-2}^{(T-1)}+\left(\beta_{T-2}^{(T-1)} \beta_{T-1}^{(T)}+\beta_{(T-2)}^{T}\right) E\left[\frac{M_{T}}{M_{T-1}} \mid \mathcal{G}_{T-1}\right]}{1+E\left[\frac{M_{T}}{M_{T-1}} \frac{\partial \eta_{T}}{\partial c_{T-1}} \mid \mathcal{G}_{T-1}\right]},
\end{aligned}
$$

where the first equation follows by (45) and the second one is due to (40). Next, by (44), (45) and (40), we have

$$
\begin{aligned}
X & :=\frac{1}{\frac{\partial \psi_{T-1}}{\partial W_{T-1}}}\left(\frac{\partial \psi_{T-1}}{\partial c_{k}}-\beta_{k}^{(T-1)}+\frac{1}{W} \frac{\partial \psi_{T-1}}{\partial W_{T-1}} \cdot \frac{\partial \eta_{T-1}}{\partial c_{k}} W\right) \\
& =-E\left[\frac{M_{T}}{M_{T-1}}\left(\frac{\partial \eta_{T}}{\partial c_{k}}+\beta_{k}^{(T-1)} \frac{\partial \eta_{T}}{\partial c_{T-1}}\right) \mid \mathcal{G}_{T-1}\right]+\frac{1}{W} \frac{\partial \eta_{T-1}}{\partial c_{k}} W \\
& =-\left(\beta_{k}^{(T-1)} \beta_{T-1}^{(T)}+\beta_{k}^{(T)}\right) E\left[\frac{M_{T}}{M_{T-1}} \mid \mathcal{G}_{T-1}\right]+\frac{1}{W} \frac{\partial \eta_{T-1}}{\partial c_{k}} W
\end{aligned}
$$


Note that the above computation implies in particular that $X \in \mathcal{L}_{T-1}$, in both cases of a deterministic interest rate and idiosyncratically incomplete markets. Indeed, in both cases $\frac{1}{W} \frac{\partial \eta_{T-1}}{\partial c_{k}} W \in \mathcal{L}_{T-1}$. In the former case, $E\left[\frac{M_{T}}{M_{T-1}} \mid \mathcal{G}_{T-1}\right]$ is a real number indicating the interest rate, and in the latter case, we have $E\left[\frac{M_{T}}{M_{T-1}} \mid \mathcal{G}_{T-1}\right]=E\left[\frac{M_{T}}{M_{T-1}} \mid \mathcal{F}_{T-1}\right] \in \mathcal{L}_{T-1}:=$ $L^{2}\left(\sigma\left(\mathcal{G}_{T-2}, \mathcal{F}_{T-1}\right)\right)$, by item (iii) in Definition 4.1, and Lemma 4.1. Therefore, by (48) and (49), we have

$$
\frac{\partial \eta_{T-1}}{\partial c_{T-2}}=-\frac{1}{\beta_{k}^{(T-2)}} X+\beta_{T-2}^{(T-1)}+\left(\beta_{T-2}^{(T-1)} \beta_{T-1}^{(T)}+\beta_{T-2}^{(T)}\right) E\left[\frac{M_{T}}{M_{T-1}} \mid \mathcal{G}_{T-1}\right] .
$$

Let us show that $E\left[-X \frac{M_{T-1}}{M_{T-2}} \mid \mathcal{G}_{T-2}\right]>0$. This will imply that

$$
\begin{aligned}
E\left[\frac{\partial \eta_{T-1}}{\partial c_{T-2}} \frac{M_{T-1}}{M_{T-2}} \mid \mathcal{G}_{T-2}\right]> & \beta_{T-2}^{(T-1)} E\left[\frac{M_{T-1}}{M_{T-2}} \mid \mathcal{G}_{T-2}\right] \\
& +\left(\beta_{T-2}^{(T-1)} \beta_{T-1}^{(T)}+\beta_{T-2}^{(T)}\right) E\left[\frac{M_{T}}{M_{T-2}} \mid \mathcal{G}_{T-2}\right] .
\end{aligned}
$$

One checks by using relation (47) that the claim is equivalent to

$$
E\left[X \frac{M_{T-1}}{M_{T-2}} \frac{\widetilde{M}_{T-2}}{\widetilde{M}_{T-1}} \frac{1}{u_{T-2}^{\prime \prime}\left(\widehat{c}_{T-2}\right)} P_{T-1}^{\mathcal{L}}\left[u_{T-1}^{\prime \prime}(Y) \frac{\partial \psi_{T-1}}{\partial W_{T-1}} X\right] \mid \mathcal{G}_{T-2}\right]>0,
$$

where,

$$
Y:=\psi_{T-1}\left(c_{0}, \ldots, c_{T-2}, W_{T-1}\right)-\sum_{k=0}^{T-2} \beta_{k}^{(T-1)} c_{k}-h_{T-1} .
$$

For a market with a deterministic interest-rate, recall that $\frac{\widetilde{M}_{k}}{M_{k}}$, is a positive number, for each $k$. Therefore, since $X \in \mathcal{L}_{T-1}$, part (iii) of Lemma 2.1 implies that (47) is equivalent to

$$
E\left[\frac{\partial \psi_{T-1}}{\partial W_{T-1}} X^{2} u_{T-1}^{\prime \prime}(Y) \mid \mathcal{G}_{T-2}\right]<0
$$

which holds true due to the assumption that $u_{T-1}$ is a concave function and that $\frac{\partial \psi_{T-1}}{\partial W_{T-1}} \geq 0$, by (44). For idiosyncratically incomplete markets, since $\frac{\widetilde{M}_{k}}{M_{k}}$ is a positive random variable, for each $k$, and the operator $P_{\mathcal{L}}^{T-1}$ is a conditional expectation, we conclude (as in (43)) that the validity of (51) is equivalent to verifying that

$$
E\left[u_{T-1}^{\prime \prime}(Y) \frac{\partial \psi_{T-1}}{\partial W_{T-1}} X^{2} \mid \sigma\left(\mathcal{G}_{T-2}, \mathcal{F}_{T-1}\right)\right]<0,
$$

which is satisfied due to the concavity of $u_{T-1}$.

The General Case. First, let us prove the statement for $k=T-2$ by exploiting the above results, and then briefly describe the general case, which is treated analogously. Consider the function

$$
\begin{aligned}
& g_{T-2}: D_{T-2} \times \mathcal{L}_{T-2} \rightarrow L^{2}\left(\mathcal{G}_{T-2}\right), \\
& g_{T-2}\left(c_{0}, \ldots, c_{T-2}, W_{T-2}\right) \\
& =c_{T-2}+E\left[\frac{M_{T-1}}{M_{T-2}} \eta_{T-1}\left(c_{0}, \ldots, c_{T-2}\right) \mid \mathcal{G}_{T-2}\right]-W_{T-2}-\epsilon_{T-2},
\end{aligned}
$$




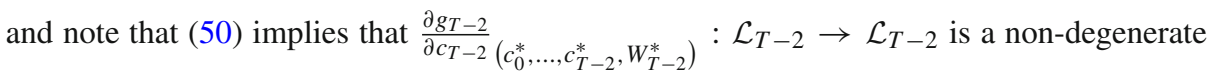
linear operator, and thus there exists a unique $C^{1}$-differentiable function $\psi_{T-2}: B_{T-2} \rightarrow$ $L^{2}\left(\mathcal{G}_{T-2}\right)$, such that

$$
g_{T-2}\left(c_{0}, \ldots, c_{T-3}, \psi_{T-2}\left(c_{0}, \ldots, c_{T-3}, W_{T-2}\right), W_{T-2}\right)=0 .
$$

Observe that a differentiation of the above equation with respect to $W_{T-2}$ combined with (50), yields

$$
\begin{aligned}
\frac{\partial \psi_{T-2}}{\partial W_{T-2}} & =\frac{1}{1+E\left[\frac{M_{T-1}}{M_{T-2}} \frac{\partial \eta_{T-1}}{\partial c_{T-2}} \mid \mathcal{G}_{T-2}\right]} \\
& \leq \frac{1}{1+\beta_{T-2}^{(T-1)} E\left[\frac{M_{T-1}}{M_{T-2}} \mid \mathcal{G}_{T-2}\right]+\left(\beta_{T-2}^{(T-1)} \beta_{T-1}^{(T)}+\beta_{T-2}^{(T)}\right) E\left[\frac{M_{T}}{M_{T-1}} \mid \mathcal{G}_{T-2}\right]}
\end{aligned}
$$

Finally, induction implies the existence of the following maps

$$
\begin{aligned}
& f_{k}: B_{k}^{\prime} \subseteq L^{2}\left(\mathcal{G}_{0}\right) \times \cdots \times L^{2}\left(\mathcal{G}_{k-1}\right) \times \mathcal{L}_{T} \rightarrow \mathcal{L}_{T}, \\
& f_{k}\left(c_{0}, \ldots, c_{k-1}, W_{k}\right)=P_{\mathcal{L}}^{k}\left[u_{k}^{\prime}\left(\psi_{k}\left(c_{0}, \ldots, c_{k-1}, W_{k}\right)-\sum_{j=0}^{k-1} \beta_{j}^{(k)} c_{j}-h_{k}\right)\right] \\
& -\frac{\widetilde{M}_{k}}{\widetilde{M}_{k-1}} u_{k-1}^{\prime}\left(\widehat{c}_{k-1}\right),
\end{aligned}
$$

for $k=1, \ldots, T-2$, where $B_{k}^{\prime}$ is the set of all tuples $\left(c_{0}, \ldots, c_{k-1}, W_{k}\right) \in B_{k}$ such that $\psi_{k}\left(c_{0}, \ldots, c_{k-1}, W_{k}\right)-\sum_{j=0}^{k-1} \beta_{j}^{(k)} c_{j}-h_{k} \geq 0$ and $\widehat{c}_{k-1} \geq 0$;

$$
\eta_{k}: D_{k-1} \subseteq L^{2}\left(\mathcal{G}_{0}\right) \times \cdots \times L^{2}\left(\mathcal{G}_{k-1}\right) \rightarrow \mathcal{L}_{k}
$$

such that

$$
f_{k}\left(c_{0}, \ldots, c_{k-1}, \eta_{k}\left(c_{0}, \ldots, c_{k-1}\right)\right)=0,
$$

for all $\left(c_{0}, \ldots, c_{k-1}\right) \in D_{k-1}, k=1, \ldots, T-1$, where $D_{k-1}$ is some open neighborhood of $\left(c_{0}^{*}, \ldots, c_{k-1}^{*}\right)$ (in the $L^{2}\left(\mathcal{G}_{0}\right) \times \cdots \times L^{2}\left(\mathcal{G}_{k-1}\right)$-topology);

$$
g_{k-1}: D_{k-1} \times \mathcal{L}_{k-1} \rightarrow L^{2}\left(\mathcal{G}_{k-1}\right)
$$

$g_{k-1}\left(c_{0}, \ldots, c_{k-1}, W_{k-1}\right)=c_{k-1}+E\left[\frac{M_{k}}{M_{k-1}} \eta_{k}\left(c_{0}, \ldots, c_{k-1}\right) \mid \mathcal{G}_{k-1}\right]-W_{k-1}-\epsilon_{k-1}$,

and

$$
\psi_{k-1}: B_{k-1} \rightarrow L^{2}\left(\mathcal{G}_{k-1}\right)
$$

such that

$$
g_{k-1}\left(c_{0}, \ldots, c_{k-2}, \psi_{k-1}\left(c_{0}, \ldots, c_{k-2}, W_{k-1}\right), W_{k-1}\right)=0,
$$

for $k=2, \ldots, T-1$, where $B_{k-1}$ is some open neighborhood of $\left(c_{0}^{*}, \ldots, c_{k-2}^{*}, W_{k-1}^{*}\right)$ in the $L^{2}\left(\mathcal{G}_{0}\right) \times \cdots \times L^{2}\left(\mathcal{G}_{k-2}\right) \times \mathcal{L}_{k-1}$-topology. In the last stage, we set

$$
g_{0}\left(c_{0}, \epsilon_{0}\right)=c_{0}+E\left[M_{1} \eta_{1}\left(c_{0}\right)\right]-\epsilon_{0},
$$


and

$$
\psi_{0}: L_{+}^{2}\left(\mathcal{G}_{0}\right) \rightarrow L_{+}^{2}\left(\mathcal{G}_{0}\right),
$$

(where $\left.L_{+}^{2}\left(\mathcal{G}_{0}\right) \cong R_{+}\right)$, such that

$$
g_{0}\left(c_{0}, \psi_{0}\left(\epsilon_{0}\right)\right)=0,
$$

for all $\epsilon_{0} \in L_{+}^{2}\left(\mathcal{G}_{0}\right)$. This completes the proof.

A further extension of the main result dealing with a characterization of the response of the wealth to consumption, for certain models of markets, is stated below.

Definition 4.2 An incomplete market is said to be of class $\mathcal{C}$, if there exists an intermediate filtration $\left(\mathcal{H}_{k}\right)_{k=1, \ldots, T}$ such that

$$
G_{k-1} \subseteq \mathcal{H}_{k} \subseteq \mathcal{G}_{k}
$$

and $P_{\mathcal{L}}^{k}[\cdot]=E\left[\cdot \mid \mathcal{H}_{t}\right]$, for all $k=1, \ldots, T$.

Theorem 4.2 For an incomplete market of type $\mathcal{C}$ with a deterministic interest rate, or for an idiosyncratically incomplete market, under the notations of Theorem 4.1, we have

$$
\frac{\partial \eta_{k+1}}{\partial c_{k}} \geq \sum_{j=k+1}^{T}\left(\sum_{i=k}^{j} \beta_{k}^{i} \beta_{i}^{i+1} \cdots \beta_{j-1}^{j}\right) E\left[\frac{M_{j}}{M_{k+1}} \mid \mathcal{G}_{k+1}\right]
$$

for all $k=0, \ldots, T-1$.

As in the proof of Theorem 4.1, our approach is applicable in both models simultaneously. We will prove the above statement for $k=T, T-1$, whereas the rest can be easily completed by induction.

Proof of Theorem 4.2 The key idea of the proof is based on the algebraic identities developed in the proof of Theorem 4.1 combined with the observation that the operators $P_{\mathcal{L}}^{k}, k=$ $1, \ldots, T$, are conditional expectations in the setting of the current theorem. Observe that identity (37) accepts the form

$$
\frac{\partial \eta_{T}}{\partial c_{T-1}}=\beta_{T-1}^{(T)}+\frac{\tilde{M}_{T}}{\widetilde{M}_{T-1}} \frac{u_{T-1}^{\prime \prime}\left(\widehat{c}_{T-1}\right)}{P_{\mathcal{L}}^{T}\left[u_{T}^{\prime \prime}\left(\psi_{T}\left(\eta_{T}\left(c_{0}, \ldots, c_{T-1}\right)\right)-\sum_{l=0}^{T-1} \beta_{l}^{(T)} c_{l}-h_{T}\right)\right]},
$$

proving the statement for $k=T$. Next, one can check by using identity (48), that (46) can be transformed into

$$
\begin{gathered}
\frac{\partial \eta_{T-1}}{\partial c_{T-2}}=\frac{1}{\frac{\partial \psi_{T-1}}{\partial W_{T-1}}\left(\beta_{T-2}^{(T-1)}-\frac{\partial \psi_{T-1}}{\partial c_{T-2}}\right)+\frac{\widetilde{M}_{T-1}}{\widetilde{M}_{T-2}}} \\
\times \frac{u_{T-2}^{\prime}\left(\widehat{c}_{T-2}\right)}{P_{\mathcal{L}}^{T-1}\left[\frac{\partial \psi_{T-1}}{\partial W_{T-1}} u_{T-1}^{\prime \prime}\left(\psi_{T-1}\left(c_{0}, \ldots, c_{T-2}, W_{T-1}\right)-\sum_{k=0}^{T-2} \beta_{k}^{(T-1)} c_{k}-h_{T-1}\right)\right]}
\end{gathered}
$$

Now, note that identities (48) and (44) guarantee the validity of the assertion for the case $k=T-1$. This completes the proof. 


\section{Concavity results in incomplete markets}

In the previous section, arbitrary time-inconsistent utility functions served as generic preference functionals, since the monotonicity feature of the consumption was valid for a large class of markets. As it is shown in the next two subsections, the concavity property of the consumption is invalid for some elementary and deterministic models of complete markets, when general preferences are involved. Therefore, we will further restrict ourselves only to some particular preferences (time-consistent power utility functions), in order to ensure the concavity property for a rich variety of financial markets (see Sect. 5.3).

\subsection{A counterexample with time-inconsistent preferences}

Consider a one-period complete market represented by the positive SPD: $M_{0}=1$ and some $M_{1}>0$. Assume that the investor is represented by the utility functions $u_{0}(x)=$ $\log x, u_{1}(x)=-x^{-1}$ and $\rho=0$.The endowments of the agent are denoted by $\epsilon_{0}$ and $\epsilon_{1}$;

the habit coefficient is assumed to be $\beta_{0}^{(1)}=1$; exogenous habits are not incorporated, i.e., $h_{1}=0$. We let $c_{0}\left(\epsilon_{0}\right)$ and $c_{1}\left(\epsilon_{0}\right)$ represent the optimal consumption stream. By Theorem 3.3, it follows that

$$
c_{1}\left(\epsilon_{0}\right)=c_{0}\left(\epsilon_{0}\right)+\sqrt{c_{0}\left(\epsilon_{0}\right) M_{1}} .
$$

The budget constraint $c_{0}\left(\epsilon_{0}\right)+E\left[M_{1} c_{1}\left(\epsilon_{0}\right)\right]=\epsilon_{0}+E\left[M_{1} \epsilon_{1}\right]$, implies that

$$
c_{0}\left(\epsilon_{0}\right)=\left(\frac{\sqrt{4 a \epsilon_{0}+4 a c+b^{2}}-b}{2 a}\right)^{2},
$$

where $a=1+E\left[M_{1}\right], b=E\left[M_{1} \sqrt{M_{1}}\right]$, and $c=E\left[M_{1} \epsilon_{1}\right]$. We have,

$$
0<c_{0}^{\prime}\left(\epsilon_{0}\right)=\frac{1}{a} \frac{\sqrt{4 a \epsilon_{0}+4 a c+b^{2}}-b}{\sqrt{4 a \epsilon_{0}+4 a c+b^{2}}}<1,
$$

and

$$
c_{0}^{\prime \prime}\left(\epsilon_{0}\right)=2 b\left(4 a \epsilon_{0}+4 a c+b^{2}\right)^{-3 / 2}>0 .
$$

Thus $c_{0}\left(\epsilon_{0}\right)$ is a convex function.

\subsection{Why only power utility functions?}

Consider a one-period complete market which consists only of a single riskless bond paying an interest rate $r \in R_{+}$. Assume that the individual is represented by the initial endowment $\epsilon_{0}>0$ and some time-consistent utility function $u: R_{+} \rightarrow R$, which satisfies the Inada conditions. We assume that no habits are involved. The corresponding utility maximization problem is:

$$
\sup _{0 \leq \pi_{0} \leq \epsilon_{0}} u\left(\epsilon_{0}-\pi_{0}\right)+u\left(\pi_{0} r\right)
$$

Let $\pi_{0}\left(\epsilon_{0}\right)$ and $c_{0}(\epsilon):=\epsilon_{0}-\pi_{0}\left(\epsilon_{0}\right)$ denote the optimal portfolio and consumption respectively. The first order conditions imply that $\pi_{0}\left(\epsilon_{0}\right)$ is determined uniquely as the solution of the equation $F\left(\epsilon_{0}, \pi_{0}\left(\epsilon_{0}\right)\right)=0$, where

$$
F\left(\epsilon_{0}, \pi_{0}\right)=r u^{\prime}\left(\pi_{0} r\right)-u^{\prime}\left(\epsilon_{0}-\pi_{0}\right),
$$


and hence, we have

$$
\pi_{0}\left(\epsilon_{0}\right)=\epsilon_{0}-I\left(r u^{\prime}\left(\pi_{0}\left(\epsilon_{0}\right) r\right)\right),
$$

where $I(x):=\left(u^{\prime}\right)^{-1}(x)$. The next statement demonstrates that only power utility functions imply the concavity of the consumption property.

Theorem 5.1 The function $c_{0}\left(\epsilon_{0}\right)$ is concave for all $r \in R_{+}$, if and only if $u(x)=c_{1}+$ $c_{2} x^{1-\gamma}$, for arbitrary $c_{1}, c_{2} \in R$ and $\gamma>0$. Furthermore, in this case,

$$
c_{0}\left(\epsilon_{0}\right)=\frac{r^{1-1 / \gamma}}{1+r^{1-1 / \gamma}} \epsilon_{0} .
$$

Therefore, the only possible form of concavity here, is linearity.

Proof of Theorem 5.1 Note that the concavity of $c_{0}\left(\epsilon_{0}\right)$ is equivalent to the convexity of $\pi_{0}\left(\epsilon_{0}\right)$. Observe that the function $\pi_{0}\left(\epsilon_{0}\right)$ is increasing, since

$$
\pi_{0}^{\prime}\left(\epsilon_{0}\right)=\frac{1}{1+r^{2} I^{\prime}\left(r u^{\prime}\left(\pi_{0}\left(\epsilon_{0}\right)\right)\right) u^{\prime \prime}\left(\pi_{0}\left(\epsilon_{0}\right) r\right)}>0 .
$$

Therefore, it follows that the function $\pi_{0}\left(\epsilon_{0}\right)$ is convex for all $r>0$, if and only if the function $h(r, x):=I\left(r u^{\prime}(r x)\right)$ is concave with respect to the variable $x$, for all $r>0$. Note that

$$
\frac{\partial h}{\partial r}(r, x)=I^{\prime}\left(r u^{\prime}(r x)\right)\left(u^{\prime}(r x)+r u^{\prime \prime}(r x) x\right) .
$$

The identity $I\left(u^{\prime}(x)\right)=x$, implies that $I^{\prime}\left(u^{\prime}(x)\right)=\frac{1}{u^{\prime \prime}(x)}$, and thus

$$
\left.\frac{\partial h}{\partial r}(r, x)\right|_{r=1}=x+\frac{u^{\prime}(x)}{u^{\prime \prime}(x)} .
$$

On the other hand, since $h(1, x)=1$, we have

$$
\left.\frac{\partial h}{\partial r}(r, x)\right|_{r=1}=\lim _{r \rightarrow 1} \frac{h(r, x)-1}{r-1} .
$$

The function $h(r, x)$ is a concave function of $x$, for all $r>0$, if and only if the function $\frac{h(r, x)-h(1, x)}{r-1}$ is a concave function of $x$, for all $r>1$, and a convex function of $x$, for all $r<1$. Therefore, it follows that $x+\frac{u^{\prime}(x)}{u^{\prime \prime}(x)}$ is a pointwise limit of concave and convex functions, and thus we have $\frac{u^{\prime}(x)}{u^{\prime \prime}(x)}=a x+b$, for some $a, b \in R$. This implies that $u^{\prime}(x)=c|a x+b|^{1 / a}$, for some $c \in R$. The Inada condition $u^{\prime}(0)=\infty$ implies that $b=0$ and $a<0$. Finally, we conclude that $u(x)=c_{1}+c_{2} x^{1-\gamma}$, for some $c_{1}, c_{2} \in R$ and $\gamma=-1 / a$, as required.

\subsection{Concavity of the optimal consumption stream}

We establish the concavity property of the optimal consumption stream for an agent whose habit-forming preference are represented by a power utility, in the setting of arbitrary idiosyncratically incomplete markets, and markets of type $\mathcal{C}$ with a deterministic interest rate (see Definition 4.2).

Theorem 5.2 Given an idiosyncratically incomplete market, or a market of type $\mathcal{C}$ with a deterministic interest rate, and an agent represented by the utility functions $u_{k}(x)=$ $e^{-\rho k} \frac{x^{1-\gamma}}{1-\gamma}$ 
$k=0, \ldots, T, \rho \geq 0, \gamma \geq 0$, the optimal consumption is a concave function of wealth.

That is, under the notations of Theorem 4.1, we have

$$
\frac{\partial^{2} \psi_{k}}{\partial^{2} W_{k}} \leq 0
$$

for all $k=1, \ldots, T-1$, and

$$
\psi_{0}^{\prime \prime}\left(\epsilon_{0}\right) \leq 0
$$

As in the proof of Theorem 4.1, we will provide a proof for the benchmark cases: $k=$ $T-1, T-2$. A proof for a general period can be carried out analogously. The proof applies to both models of markets.

Proof of Theorem 5.2 A differentiation of Eq. 44 with respect to $W_{T-1}$, yields

$$
\frac{\partial^{2} \psi_{T-1}}{\partial^{2} W_{T-1}}=-\frac{1}{1+\left(E\left[\frac{M_{T}}{M_{T-1}} \frac{\partial \eta_{T}}{\partial c_{T-1}} \mid \mathcal{G}_{T-1}\right]\right)^{2}} E\left[\frac{M_{T}}{M_{T-1}} \frac{\partial^{2} \eta_{T}}{\partial^{2} c_{T-1}} \frac{\partial \psi_{T-1}}{\partial W_{T-1}} \mid \mathcal{G}_{T-1}\right] .
$$

By differentiating Eq. 58 with respect to $c_{T-1}$, we get

$$
\frac{\partial^{2} \eta_{T}}{\partial^{2} c_{T-1}}=\frac{\widetilde{M}_{T}}{\widetilde{M}_{T-1}} \frac{u_{T-1}^{\prime \prime \prime}(X) P_{\mathcal{L}}^{T}\left[u_{T}^{\prime \prime}(Y)\right]-u_{T-1}^{\prime \prime}(X) P_{\mathcal{L}}^{T}\left[u_{T}^{\prime \prime}(Y)\right]\left(\frac{\partial \eta_{T}}{\partial c_{T-1}}-\beta_{T-1}^{(T)}\right)}{\left(P_{\mathcal{L}}^{T}\left[u_{T}^{\prime \prime}(Y)\right]\right)^{2}},
$$

where $X:=\widehat{c}_{T-1}$, and

$$
Y:=\psi_{T}\left(\eta_{T}\left(c_{0}, \ldots, c_{T-1}\right)\right)-\sum_{l=0}^{T-1} \beta_{l}^{(T)} c_{l}-h_{T} .
$$

Observe that we have explicitly exploited the fact that $P_{\mathcal{L}}^{T}[\cdot]=E\left[\cdot \mid \mathcal{H}_{T-1}\right]$ for markets of type $\mathcal{C}$, and $P_{\mathcal{L}}^{T}[\cdot]=E\left[\cdot \mid \sigma\left(\mathcal{G}_{T-1}, \mathcal{F}_{T}\right)\right]$ for idiosyncratically incomplete markets, by taking the term $\left(\frac{\partial \eta_{T}}{\partial c_{T-1}}-\beta_{T-1}^{(T)}\right)$ out of the brackets. Furthermore, recall that $\frac{\partial \psi_{T-1}}{\partial W_{T-1}} \geq 0$ by Theorem 4.1, and note that $M_{k} \widetilde{M}_{k} \geq 0, k=1, \ldots, T$, since $\frac{M_{k}}{\widetilde{M}_{k}} \geq 0$ in the setting of both markets. Consequently, in order to prove the statement for $k=T-1$, it suffices to check that

$$
u_{T-1}^{\prime \prime \prime}(X) P_{\mathcal{L}}^{T}\left[u_{T}^{\prime \prime}(Y)\right]-u_{T-1}^{\prime \prime}(X) P_{\mathcal{L}}^{T}\left[u_{T}^{\prime \prime}(Y)\right]\left(\frac{\partial \eta_{T}}{\partial c_{T-1}}-\beta_{T-1}^{(T)}\right) \geq 0,
$$

which is, by (37), equivalent to

$$
\left(P_{\mathcal{L}}^{T}\left[Y^{-1-\gamma}\right]\right)^{2} \leq X^{-\gamma} P_{\mathcal{L}}^{T}\left[Y^{-2-\gamma}\right] \frac{\widetilde{M}_{T}}{\widetilde{M}_{T-1}} .
$$

Notice now that Lemma 4.2 for $k=T$, implies that the above inequality can be rewritten as

$$
\left(P_{\mathcal{L}}^{T}\left[Y^{-1-\gamma}\right]\right)^{2} \leq P_{\mathcal{L}}^{T}\left[Y^{-2-\gamma}\right] P_{\mathcal{L}}^{T}\left[Y^{-\gamma}\right]
$$


which, by the fact that $P_{\mathcal{L}}^{T}[\cdot]$ is a conditional expectation in the setting of both markets, is satisfied by the Cauchy-Schwartz inequality. Next, let us treat the case: $k=T-2$. A differentiation of Eq. 52 with respect to $W_{T-2}$ yields

$$
\frac{\partial^{2} \psi_{T-2}}{\partial^{2} W_{T-2}}=-\frac{1}{\left(1+E\left[\frac{M_{T-1}}{M_{T-2}} \frac{\partial \eta_{T-1}}{\partial c_{T-2}} \mid \mathcal{G}_{T-2}\right]\right)^{2}} E\left[\frac{M_{T-1}}{M_{T-2}} \frac{\partial^{2} \eta_{T-1}}{\partial^{2} c_{T-2}} \frac{\partial \psi_{T-2}}{\partial W_{T-2}} \mid \mathcal{G}_{T-2}\right],
$$

and by differentiating Eq. 59 with respect to $c_{T-2}$, we obtain that

$$
\begin{aligned}
& \frac{\partial^{2} \eta_{T-1}}{\partial^{2} c_{T-2}}=\frac{\tilde{M}_{T-1}}{\widetilde{M}_{T-2}} \frac{1}{\left(P_{\mathcal{L}}^{T-1}\left[u_{T-1}^{\prime \prime}\left(\widehat{c}_{T-1}\right) \frac{\partial \psi_{T-1}}{\partial W_{T-1}}\right]\right)^{2}} \\
& \times\left(u_{T-2}^{\prime \prime \prime}\left(\widehat{c}_{T-2}\right) P_{\mathcal{L}}^{T-1}\left[u_{T-1}^{\prime \prime}\left(\widehat{c}_{T-1}\right) \frac{\partial \psi_{T-1}}{\partial W_{T-1}}\right]\right. \\
& -u_{T-2}^{\prime \prime}\left(\widehat{c}_{T-2}\right) P_{\mathcal{L}}^{T-1}\left[u_{T-1}^{\prime \prime \prime}\left(\widehat{c}_{T-1}\right)\left(\frac{\partial \psi_{T-1}}{\partial c_{T-2}}+\frac{\partial \psi_{T-1}}{\partial W_{T-1}} \frac{\partial \eta_{T-1}}{\partial c_{T-2}}-\beta_{T-2}^{(T-1)}\right) \frac{\partial \psi_{T-1}}{\partial W_{T-1}}\right] \\
& \left.-u_{T-2}^{\prime \prime}\left(\widehat{c}_{T-2}\right) P_{\mathcal{L}}^{T-1}\left[u_{T-1}^{\prime \prime \prime}\left(\widehat{c}_{T-1}\right)\left(\frac{\partial^{2} \psi_{T-1}}{\partial^{2} W_{T-1}} \frac{\partial \eta_{T-1}}{\partial c_{T-2}}+\frac{\partial^{2} \psi_{T-1}}{\partial W_{T-1} \partial c_{T-2}}\right)\right]\right) .
\end{aligned}
$$

We shall now simplify the above expression. By plugging (44) into (48), and by differentiating the latter equation with respect to $W_{T-1}$, we obtain that

$$
\frac{\partial^{2} \psi_{T-1}}{\partial c_{T-2} \partial W_{T-1}}=-\frac{\partial^{2} \psi_{T-1}}{\partial^{2} W_{T-1}}\left(\beta_{T-2}^{(T-1)}+E\left[\left(\beta_{T-2}^{(T-1)} \frac{\partial \eta_{T}}{\partial c_{T-1}}+\frac{\partial \eta_{T}}{\partial c_{T-2}}\right) \frac{M_{T}}{M_{T-1}} \mid \mathcal{G}_{T-1}\right]\right) .
$$

Therefore, we get

$$
\begin{aligned}
& \frac{\partial^{2} \psi_{T-1}}{\partial^{2} W_{T-1}} \frac{\partial \eta_{T-1}}{\partial c_{T-2}}+\frac{\partial^{2} \psi_{T-1}}{\partial W_{T-1} \partial c_{T-2}} \\
& \quad=\frac{\partial^{2} \psi_{T-1}}{\partial^{2} W_{T-1}}\left(\frac{\partial \eta_{T-1}}{\partial c_{T-2}}-\beta_{T-2}^{(T-1)}-E\left[\left(\beta_{T-2}^{(T-1)} \frac{\partial \eta_{T}}{\partial c_{T-1}}+\frac{\partial \eta_{T}}{\partial c_{T-2}}\right) \frac{M_{T}}{M_{T-1}} \mid \mathcal{G}_{T-1}\right]\right) \\
& \quad=\frac{\partial^{2} \psi_{T-1}}{\partial^{2} W_{T-1}}\left(\frac{\widetilde{M}_{T-1}}{\widetilde{M}_{T-2}} \frac{u_{T-2}^{\prime \prime}\left(\widehat{c}_{T-2}\right)}{P_{\mathcal{L}}^{T-1}\left[u_{T-1}^{\prime \prime}\left(\widehat{c}_{T-1}\right) \frac{\partial \psi_{T-1}}{\partial W_{T-1}}\right]}\right),
\end{aligned}
$$

where the last equality follows by combining relations (48), (44) and (59). Next, by (59), it follows that

$$
\begin{aligned}
& P_{\mathcal{L}}^{T-1}\left[u_{T-1}^{\prime \prime \prime}\left(\widehat{c}_{T-1}\right)\left(\frac{\partial \psi_{T-1}}{\partial c_{T-2}}+\frac{\partial \psi_{T-1}}{\partial W_{T-1}} \frac{\partial \eta_{T-1}}{\partial c_{T-2}}-\beta_{T-2}^{(T-1)}\right) \frac{\partial \psi_{T-1}}{\partial W_{T-1}}\right] \\
& \quad=P_{\mathcal{L}}^{T-1}\left[u_{T-1}^{\prime \prime \prime}\left(\widehat{c}_{T-1}\right)\left(\frac{\partial \psi_{T-1}}{\partial W_{T-1}}\right)^{2} \frac{\widetilde{M}_{T-1}}{\widetilde{M}_{T-2}} \frac{u_{T-2}^{\prime \prime}\left(\widehat{c}_{T-2}\right)}{P_{\mathcal{L}}^{T-1}\left[u_{T-1}^{\prime \prime}\left(\widehat{c}_{T-1}\right) \frac{\partial \psi_{T-1}}{\partial W_{T-1}}\right]}\right] .
\end{aligned}
$$

Finally, by combining the above observations, it follows that in order to show that $\frac{\partial^{2} \psi_{T-2}}{\partial^{2} W_{T-2}} \leq 0$, it suffices to check that

$$
X^{-2-\gamma} P_{\mathcal{L}}^{T-1}\left[Y^{-1-\gamma} \frac{\partial \psi_{T-1}}{\partial W_{T-1}}\right]-X^{-2-\gamma} \frac{\widetilde{M}_{T-1}}{\widetilde{M}_{T-2}} P_{\mathcal{L}}^{T-1} \frac{\left[Y^{-2-\gamma}\left(\frac{\partial \psi_{T-1}}{\partial W_{T-1}}\right)^{2}\right]}{\left[Y^{-1-\gamma} \frac{\partial \psi_{T-1}}{\partial W_{T-1}}\right]} \leq 0,
$$


where $X:=\widehat{c}_{T-1}$ and $Y:=\widehat{c}_{T-2}$. By Lemma 4.2 for $k=T-1$, the latter inequality is equivalent to

$$
X^{-2-\gamma} P_{\mathcal{L}}^{T-1}\left[Y^{-1-\gamma} \frac{\partial \psi_{T-1}}{\partial W_{T-1}}\right]-X^{-2-2 \gamma} \frac{P_{\mathcal{L}}^{T-1}\left[Y^{-\gamma}\right]}{X^{-\gamma}} \frac{P_{\mathcal{L}}^{T-1}\left[Y^{-2-\gamma}\left(\frac{\partial \psi_{T-1}}{\partial W_{T-1}}\right)^{2}\right]}{P_{\mathcal{L}}^{T-1}\left[Y^{-1-\gamma} \frac{\partial \psi_{T-1}}{\partial W_{T-1}}\right]} \leq 0,
$$

or equivalently

$$
\left(P_{\mathcal{L}}^{T-1}\left[Y^{-1-\gamma} \frac{\partial \psi_{T-1}}{\partial W_{T-1}}\right]\right)^{2} \leq P_{\mathcal{L}}^{T-1}\left[Y^{-\gamma}\right] P_{\mathcal{L}}^{T-1}\left[Y^{-2-\gamma}\left(\frac{\partial \psi_{T-1}}{\partial W_{T-1}}\right)^{2}\right],
$$

which is satisfied by the Cauchy-Schwartz inequality, since the projection $P_{\mathcal{L}}^{T-1}$ is a conditional expectation in both settings of markets.

Acknowledgements I am grateful to my supervisor Semyon Malamud for very helpful discussions, and for detailed comments on the preliminary version of the manuscript. I would also like to thank an anonymous referee for useful remarks. Financial support by the Swiss National Science Foundation via the SNF Grant PDFM2-120424/1 is gratefully acknowledged.

\section{References}

1. Abel, A.: Asset prices under habit formation and catching up with the Joneses. Am. Econ. Rev. 80, 38$42(1990)$

2. Campbell, J.Y., Cochrane, J.: By force of habit: a consumption-based explanation of aggregate stock market behavior. J. Polit. Econ. 107, 205-251 (1999)

3. Carroll, C., Kimball, M.: On the concavity of the consumption function. Econometrica 64(4), 981992 (1996)

4. Chan, Y., Kogan, L.: Catching up with the Joneses: heterogeneous preferences and the dynamics of asset prices. J. Polit. Econ. 110, 1255-1285 (2002)

5. Chapman, D.A.: Habit formation and aggregate consumption. Econometrica 66(5), 1223-1230 (1998)

6. Constantinides, G.M.: Habit formation: a resolution of the equity premium puzzle. J. Polit. Econ. 98(3), 519-543 (1990)

7. Dalang, R., Morton, A., Willinger, W.: Equivalent martingale measures and no-arbitrage in stochastic securities market models. Stoch. Stoch. Rep. 29(2), 185-201 (1990)

8. Detemple, J., Karatzas, I.: Non-addictive habits: optimal consumption-portfolio policies. J. Econ. Theory 113, 265-285 (2003)

9. Detemple, J., Zapatero, F.: Asset prices in an exchange economy with habit formation. Econometrica c59, 1633-1657 (1991)

10. Detemple, J., Zapatero, F.: Optimal consumption-portfolio policies with habit formation. Math. Finan. 2(4), 251-274 (1992)

11. Duffie, D.: Dynamic Asset Pricing Theory. Princeton University Press, Princeton (2001)

12. Duffie, D., Fleming, W., Soner, H.M., Zariphopoulou, T.: Hedging in incomplete markets with HARA utility. J. Econ. Dyn. Control 21, 753-781 (1997)

13. Englezos, N., Karatzas, I.: Utility maximization with habit formation: dynamic programming and stochastic PDEs. SIAM J. Control Optim. 48(2), 481-520 (2009)

14. Ekeland, I., Lazrak, A.: The golden rule when preferences are time inconsistent. Math. Finan. Econ. 4, 29-55 (2010)

15. Gomes, F., Michaelides, A.: Portfolio choice with internal habit formation: a life-cycle model with uninsurable labor income risk. Rev. Econ. Dynam. 6(4), 729-766 (2003)

16. Heaton, J.: Consumption and portfolio policies with incomplete markets and short-sale constraints: the infinite-dimensional case. Econometrica 61(2), 353-385 (1993)

17. He, H., Pearson, N.D.: Consumption and portfolio policies with incomplete markets and short-sale constraints: the infinite-dimensional case. Math. Finan. 1, 1-10 (1991)

18. He, H., Pearson, N.D.: Consumption and portfolio policies with incomplete markets and short-sale constraints: the infinite-dimensional case. J. Econ. Theory 54, 259-304 (1991) 
19. Hendersen, V.: Explicit solutions to an optimal portfolio choice problem with stochastic income. J. Econ. Dyn. Control. 29(7), 1237-1266 (2005)

20. Karatzas, I., Lehoczky, J.P., Shreve, S.E., Xu, G.L.: Martingale and duality methods for utility maximisation in an incomplete market. SIAM J. Control Optim. 29, 702-730 (1991)

21. Karatzas, I., Žitković , G.: Optimal consumption from investment and random endowment in incomplete semimartingale markets. Ann. Probab. 31(4), 1821-1858 (2003)

22. Karp, L.: Non-constant discounting in continuous time. J. Econ. Theory 132, 557-568 (2007)

23. Keynes, J.M.: The General Theory of Employment, Interest and Money. Cambridge University Press, Cambridge (1936)

24. Malamud, S.: Asset pricing for idiosyncratically incomplete markets. PhD Thesis, ETH Zurich, Diss. ETH No. 16651 (2006)

25. Malamud, S., Trubowitz, E.: The structure of optimal consumption streams in general incomplete markets. Math. Finan. Econ. 1, 129-161 (2007)

26. Merton, R.: Optimum consumption and portfolio rules in a continuous time model. J. Econ. Theory 3(4), 373-413 (1971)

27. Pliska, S.: Introduction to Mathematical Finance: Discrete Time Models. Blackwell, Malden (1997)

28. Plott, C.: Rational choice in experimental markets. J. Bus. 59, S301-S327 (1986)

29. Polkovnichenko, V.: Life-cycle portfolio choice with additive habit formation preferences and uninsurable labor income risk. Rev. Finan. Stud. 20(1), 83-124 (2007)

30. Rásonyi, M., Stettner, Ł.: On utility maximization in discrete-time market models. Ann. Appl. Probab. 15, 1367-1395 (2005)

31. Taqqu, M.S., Willinger, W.: The analysis of finite security markets using martingales. Adv. Appl. Probab. 19, 1-25 (1987)

32. Zariphopoulou, T.: Optimal asset allocation in a stochastic factor model-an overview and open problems. Adv. Finan. Model. Radon Ser. Comp. Appl. Math 8, 427-453 (2009) 